Rhode Island College

Digital Commons @ RIC

Master's Theses, Dissertations, Graduate

Master's Theses, Dissertations, Graduate Research and Major Papers Overview

Research and Major Papers

$5-10-2020$

\title{
Exploring Nurses' Knowledge of Medication Error Reporting
}

Karina Santos

Follow this and additional works at: https://digitalcommons.ric.edu/etd

Part of the Nursing Commons

\section{Recommended Citation}

Santos, Karina, "Exploring Nurses' Knowledge of Medication Error Reporting" (2020). Master's Theses, Dissertations, Graduate Research and Major Papers Overview. 365.

https://digitalcommons.ric.edu/etd/365

This Major Paper is brought to you for free and open access by the Master's Theses, Dissertations, Graduate Research and Major Papers at Digital Commons @ RIC. It has been accepted for inclusion in Master's Theses, Dissertations, Graduate Research and Major Papers Overview by an authorized administrator of Digital Commons @ RIC. For more information, please contact digitalcommons@ric.edu. 


\section{EXPLORING NURSES' KNOWLEDGE OF MEDICATION ERROR}

\section{REPORTING IN THE CRITICAL CARE SETTING}

by

Karina Santos, BSN, RN

A Major Paper Submitted in Partial Fulfillment of the Requirements for the Degree of

Master of Science in Nursing

in

The School of Nursing

Rhode Island College

2020 


\begin{abstract}
Managing the care of critically ill patients is a highly complex and stressful position requiring high levels of critical thinking skills and judgment. Medical errors, including medication errors continue to happen in hospitals across the nation. Despite decades of focus and efforts on this area from the Institute of Medicine and other national and federal agencies, literature suggests that medication errors in critical care are highly prevalent and underreported. The purpose of this project was to explore the knowledge base of critical care nurses in relation to medication error reporting. A survey was created, which included 10 questions that were relevant to medication errors and reporting of these in the critical care setting. No demographical data was collected on respondent nurses to protect anonymity and privacy. A total of 77 completed surveys were collected from ten critical care units at a large academic acute care hospital in Rhode Island. The results of the survey showed that the majority of nurses had no knowledge of the hospital's policy or the official definition of a medication error as adopted by the facility. A significant number of nurses weren't aware that near miss events are medication errors. This project found that critical care nurses and their patients would benefit from enhanced education programs aimed at closing these knowledge gaps. Providing clarification, guidelines and detailed policies and procedures may enhance their confidence, efficacy and skills to be able to adequately and consistently report all near miss events and actual medication errors thereby improving the overall culture of safety and patient outcomes.
\end{abstract}

Keywords medical error, medication error, reporting medication error, adverse drug events, patient safety, critical care, medication safety 


\section{Acknowledgements}

I want to acknowledge the staff at Rhode Island College and Rhode Island Hospital who have mentored me over many years. All my professors, fellow students, senior nurses, and preceptors as well as physicians, therapists and other healthcare professionals who have taught me and nurtured all the knowledge I have acquired over a thirteen-year career. I am proud to call you colleagues, friends and family.

This paper is especially dedicated to those who serve on the front line. I am proud to serve alongside all of you. Those whom have stepped up and worked tirelessly through the most devastating public health crisis in our lifetime. The nursing profession stands hand in hand with the world our shoulders dedicated to each other, our communities, our patients and our work. This is our moment in history, and we will rise to the challenge. Now, with this project completed, I join the ranks alongside you all at the frontline where I belong.

To my daughter Roslyn, I know you will see many challenges in your lifetime. I hope I have set the best example for you to meet those challenges head on with gut and grit. Persevere my sweet girl, succeed and fight above all for what you think is right. At your very young age you have also sacrificed and had to share me with my "honeys", my work and my studies. You selflessly stood by as I pursued my dream and tried to help others. You have given up story-time, snuggles and kisses for the greater good. I want you to know you and your brother are my ultimate inspiration for all I do. You inspire me to be better and to make this world as safe for you as I can. I hope I've made you proud. 


\section{Table of Contents}

Background/Statement of the Problem .......................................................... 1

Literature Review.......................................................... 4

Theoretical Framework.......................................................... 26

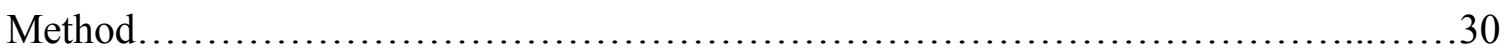

Results.................................................................... 35

Summary and Conclusions..............................................45

Recommendations and Implications for Advanced Nursing Practice...................51

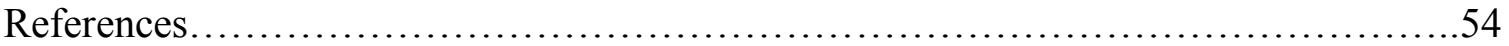

Appendices................................................................ 71 


\section{Exploring Nurses’ Knowledge of Medication Error Reporting in the Critical Care Setting}

\section{Background/Statement of the Problem}

A local newspaper reported the death of a woman in an Oregon hospital in 2014. Doctors prescribed an intravenous antiseizure medication but instead the patient received a dose of a neuromuscular blocking agent resulting in cardiac arrest and her death days later (Bannow, 2014, para. 1). In 2017, a patient in a Tennessee Hospital was given the same neuromuscular blocking agent in error. An intravenous sedative was prescribed to help with anxiety while undergoing diagnostic imaging, but the incorrect medication was administered, also resulting in death (Kelman, 2019, para. 1). The nurse involved in the latter incident was indicted on charges of reckless homicide in early 2019.

Almost twenty years ago, the Institute of Medicine (IOM) published its report, To Err is Human, estimating up to 98,000 annual deaths as a result of medical errors (Kohn, Corrigan, \& Donaldson, 1999). In 2016, a John Hopkins study reported an updated estimate of 134,581 deaths in hospitals each year due to medical error. These new figures would make medical error the third leading cause of death in the nation (Makary \& Daniel, 2016).

Medication errors are one of the most common type of medical error (Kohn et al., 1999). In 2010, 31\% of adverse events (AEs) found were related to medications and seven of twelve lethal AEs studied were attributed to medications and deemed adverse drug events (Landrigan et al., 2010). Adverse drug event (ADE) rates have been reported as $33.7 \%$ per 1,000 discharges in 2014 . Preliminary data from 2017 reveals 721,000 ADEs or $24.2 \%$ per 1,000 discharges. The estimated extra cost of care related to ADEs is 
estimated at $\$ 5,746$ as of 2017 up from $\$ 5,452$ in 2010 (Agency for Healthcare Research and Quality [AHRQ], 2018).

Patients admitted to critical care units carry higher levels of morbidity and mortality leading to a higher susceptibility to harm from medication errors. In a study by Rothschild et al. (2005), AE rates in critical care units were reported as high as $80.5 \%$ per 1,000 patient-days. Medications were found to be responsible for $61 \%$ of most serious, life-threatening adverse events (AE) in critical care (Rothschild et al., 2005), and 23\% of preventable ADEs took place at the administration stage of the medication use process (Kopp, Erstad, Allen, Theodorou, \& Priestly, 2006). Intravenous (IV) medications carry a higher risk for error with a reported $49 \%$ of IV medication administration doses containing at least one error (Taxis \& Barber, 2003). Nurses are unavoidably responsible for the administration of medications and therapies in critical care or intensive care units (ICUs) and thus are more likely to experience medication administration errors (MAEs). Event or incident reporting is crucial in reducing medical and medication errors using a systems approach. Incident reporting rates are highest amongst nurses and yet only one in seven medical errors were found to be reported (Levinson, 2010). Underreporting of medical and medication errors has been documented in the literature (Bayazidi, Zarezadeh, Zamanzadeh, \& Parvan, 2012; Hajibabaee, Joolaee, Peyravi, Alijany-Renany, \& Bahrani, 2014; Soydemir, Intepeler, \& Mert, 2016). Data on patient safety in the ICU setting is varied and limited by heterogeneity of settings, population demographics, varying detection methods and a general underreporting of medication errors and near-miss events. There is a need for updated and more robust data on adverse and near miss events in critical care units. This information can only come from reporting 
by those working on the frontline. Critical care nurses are faced with many barriers to completing incident reports regarding medication errors. One of these barriers which contributes to underreporting is a lack of education and/or knowledge regarding medication errors and the reporting process.

The purpose of this project was to explore the knowledge base of critical care nurses in relation to medication error reporting. This information was then be used for identifying knowledge gaps and needed enhanced education. 


\section{Literature Review}

Utilizing the electronic databases of PubMed, CINAHL, Ovid and Google

Scholar, a comprehensive literature search was conducted. The search terms and combination of search terms included: hospital, nurses or nursing, medication errors or medication administration errors (ME or MAE), adverse drug event, critical care or ICU, reporting, medication error reporting (MER), incident reporting, culture of safety and patient safety and quality. The search produced a vast array of articles which were reviewed and analyzed for content. Those included in the review of the literature were selected based on their relevance to medication error reporting in the hospital setting. Articles included in this review were limited to include studies on adult populations, acute hospital settings, publication between 2000 and 2019 and were written in or translated to English. Relevant studies conducted abroad or seminal research prior to 2000 are identified as such in the review.

\section{Errors in Healthcare}

Research into human error and its role in the cause of adverse events in healthcare had its beginning in the 1990's. A few pioneer researchers took on the task of investigating, defining and understanding how and why errors occurred in healthcare systems, mainly in the hospital setting. One of the leading researchers on human error, James Reason, is also an advocate of a systems approach to errors. Throughout his work, he was influenced by the work on human factors by Jens Rasmussen (Reason, 1990). Reason is the developer of the now commonly known "Swiss Cheese Model of Medical Errors," which he used to illustrate how smaller errors and system flaws can lead to bigger catastrophes. His work also introduced various other concepts that support a systems approach to managing errors. Reason introduced the terms "active" errors which 
involve front line workers and "latent" errors that are failures of design or organization. He built on these concepts to shape the terms "sharp end" to correspond to active errors involving frontline staff and "blunt end" to correspond with latent errors involving system failures. The main focus of Reason's work was to emphasize that human error is inevitable but can be mitigated using an approach that does not focus on or punish individuals who make mistakes but rather one that expects errors and creates a system that seeks to catch these and prevent harm (Reason, 2000).

Leape (1994) relied heavily on the work regarding systems and human factors by Reason and Rasmussen and defined errors as variations in processes rather than failures of an individual. He also described total quality management as "a culture in which errors and deviations are regarded not as human failures but as opportunities to improve the system" (Leape, 1994, p. 1857). The IOM (now the Health and Medicine Division of the National Academies of Sciences, Engineering and Medicine) based the recommendations in To Err is Human on the work in the 1990's by Leape, Reason and Rasmussen among others (Kohn et al., 1999).

Medical error is defined as "the failure of a planned action to be completed as intended or the use of a wrong plan to achieve an aim," (Kohn et al., 1999, p. 4). An adverse event (AE) is defined as "an injury resulting from medical intervention, not an underlying condition," (Kohn et al., 1999, p. 4).

The Joint Commission (previously the Joint Commission on Accreditation of Healthcare Organizations or JCAHO) adopted a sentinel event policy in 1996 and lists reportable events as either a patient safety event, adverse event, sentinel event, close call or hazardous condition. According to the Joint Commission a sentinel event is defined as 
"a patient safety event that reaches a patient and results in any of the following: death, permanent harm or severe temporary harm and intervention required to sustain life" (The Joint Commission [TJC], 2019, "Sentinel Events Policy and Procedures," para.2). The National Quality Forum adopted a list of serious reportable events in 2002, including surgical, product, patient protection, care management, environmental and criminal events. One serious reportable care management event would include a "patient death or serious disability associated with a medication error" (National Quality Forum [NQF], 2011, p. 9).

It has been estimated that rates of adverse events range from $2.9-16.6 \%$ per 1,000 hospital inpatient admission (Milch et al., 2005; Murff, Patel, Hripcsak \& Bates, 2003). The Centers for Medicare and Medicaid (CMS) were involved in two inquiries into adverse events suffered by beneficiaries in US hospitals via the Office of the Inspector General (OIG) in 2008 and 2010 (Levinson, 2008). The 2008 study found high rates of AEs in two counties in the same state. It also brought attention to the lack of homogeneity among care settings regarding definitions and variability of what was considered an AE and if these should be reported. Based on the findings in 2008, the OIG followed up in 2010 and found 13.5\% (1 in 7) of Medicare patients were reported to suffer an adverse event while hospitalized (Levinson, 2010).

Part of the reason for the difficulty in assessing incidence, costs and causes of medical errors including medication errors is the lack of consistency in methods across healthcare organizations to identify and report AEs. A retrospective study of 10 North Carolina hospitals also looked at harm from medical care and found 588 incidences of harm in 2,341 admissions using the Institute for Healthcare Improvement's Global 
Trigger Tool for Measuring Adverse Events from 2002-2007 (Landrigan et al., 2010). In this study, it was reported that $63 \%$ of lethal AE's were attributed to medical errors. Retrospective chart reviews were conducted using the triggers to identify events that require further investigation. For example, the order or administration of an antidote medication such as Naloxone would trigger an event review. Classen et al., (2011) found that this tool was shown to have an increased ability to detect AEs than other methods in the past. This method resulted in data that was more reliable than previous works with findings of AEs in one third of hospital admissions.

It is important to note that a medical error does not necessarily result in an adverse event or patient harm, just as an adverse event does not necessarily indicate that an error took place. Studies have looked at various methods of identifying adverse events and medical errors including voluntary reporting, chart reviews and computerized monitoring and compared them for feasibility, cost and efficiency (Gandhi, Seger, \& Bates, 2000). The most effective and practical method to detect medical errors has been direct observation or a mixed methods approach that includes direct observation, although these tend to be costly and time consuming (Flynn, Barker, Pepper, Bates, \&Mikeal, 2002; Ghandi et al., 2000; Murff, Patel, Hripcsak, \& Bates, 2003).

\section{Medication Errors}

A medication error is a type of medical error. The National Coordinating Council for Medication Error Reporting and Prevention defines a medication error as "any preventable event that may cause or lead to inappropriate medication use or patient harm while in the medication is in the control of the health care professional, patient, or 
consumer" (National Coordinating Council for Medication Error Reporting and Prevention [NCCMERP], 2015, p. 2).

An ADE is defined as "an injury from medical intervention related to a drug" (Aspden, Wolcott, Bootman, \& Cronenwett, 2007, p. 4). The severity of a medication error has historically been based on patient harm. An ADE could result in a patient safety event that is reportable to TJC. If the patient experiences anaphylaxis, whether the patient recovers or the reaction leads to death, it would be a sentinel event. The committee that authored, To Err is Human, had a very clear focus on medication errors as they have been found to be one of the most common medical errors (Kohn, Corrigan, \& Donaldson, 1999). In their investigations, medication errors of all types were found to be responsible for "one out of 854 inpatient deaths" (Kohn et al., 1999, p. 27).

Medication errors (ME) can happen through the spectrum of the medication management process and do not necessarily lead to an ADE. Levinson (2010) reports that $31 \%$ of AEs were found to be ADEs in the OIG study. Reports included twelve ADEs contributing to the patient's death; seven of the twelve ADEs were related to medication administration and/or management (Levinson, 2010). Variability also exists across settings and hospitals as to what is considered a clinically significant medication error and what is a significant ADE. The IOM in 2007 again, took on the task of tackling the problem. The committee put forth a comprehensive review of current climate surrounding medication errors and made recommendations for reducing the incidence of medication errors (Aspden et al., 2007).

A systematic review reported rates of ADEs in hospital admissions that varied between $5.1-12.8 \%$, however it is difficult to discern from methods used in this study 
whether ADEs stemmed from medication errors. Causes of medication errors whether or not they lead to an ADE are varied as well but a majority of medication errors are caused by "slips and lapses" (Keers, Williams, Cooke, \& Ashcroft, 2013). Milch et al. (2005) looked are reports from 26 random and varied U.S. hospital settings to find 33\% of ADEs reported were medication or infusion related. Nebeker, Hoffman, Weir, Bennett and Hurdle (2005) found one quarter of study admissions involved at least one ADE and 27\% of those ADEs were attributed to medication errors.

The past two decades have seen some advances in the area of patient safety. The IOM's 2001 report, Crossing the Quality Chasm, cited the "growing complexity of science and technology, resulting from the tremendous advances made in clinical knowledge, drugs, medical devices, and technologies for use in patient care, as one of the four main attributes of the U.S. health system affecting health care quality" (Institute of Medicine [IOM], 2001, para. 3). The integration of computerized physician order entry (CPOE), the electronic health record (EHR) along with an electronic medication administration record (eMAR) have reduced overall rates of medication errors. When these were combined with barcode assisted medication administration (BCMA) and smart infusion pumps, there was a real opportunity to curtail most medication errors (Poon et al, 2010; Truitt et al., 2016; Keane, 2014; Wiseman, 2018). A survey by the American Society of Hospital Pharmacists (ASHP) on the integration of technology for medication use reveals the drastic changes in medication management. One survey was conducted in 2007 and a second most recently in 2013 and both surveyed hospitals across the US (Fox, Pedersen, \& Gumpper, 2015). When compared to the results of the 2007 survey, in 2013 the adoption of EHR was 33\%, up from 5.9\% in 2007, CPOE use was reported at over 
$75 \%$ of hospitals surveyed compared to $17.8 \%$, automated dispensing cabinet use increased to $61.4 \%$ from $12 \%$ and BCMA rose to $75 \%$ from $24.1 \%$. A combined effort of using eMAR with BCMA and integrated nursing documentation was reported in $60 \%$ of hospitals in the 2013 survey from 19.6\% (2007) and use of clinical decision support for CPOE programs increased to more than 60\% (2013) from 12\%when compared to 2007 survey. The results demonstrate a serious investment and commitment from hospital organizations to reducing medication errors nationwide.

\section{Medication Safety in Critical Care}

Critical care units or intensive care units (ICU) are a fast paced, highly technical and stressful work environment with many high-risk and life-saving interventions. Critically ill patients are a particularly vulnerable population due to their severe illness requiring highly complex care. Patients in ICU may receive numerous therapies and/or medications at any given time depending on their condition. The majority of medication errors that take place in critical care units are considered serious and/or life threatening (Moyen \& Stelfox, 2008). When compared to other areas, critical care/ICU medication errors accounted for $6.6 \%$ of errors reported to a national error database between 1999 and 2005. Medication errors in ICUs were more likely to be associated with harm, permanent harm, harm requiring life-sustaining interventions, or death at a rate of $3.7 \%$ reported in a national study (Latif, Rawat, Pustavoitau, Pronovost, \& Pham, 2013).

A gap exists in the research regarding medication errors in critical care/ ICU setting with most reliable research in the United States being conducted in the 1990's and published in the early 2000's. In the past two decades, critical care has become more 
complex and even more technological with implementation of health IT and further advancement of health-related technologies and medical devices.

A large, multinational study used self-reporting methods by ICU staff over a 24hour period to assess medication errors in parenteral drug administration. The participating 113 ICUs were spread over 27 countries and five continents. The study found 861 medication errors that affected 441 patients. Twelve of the 441 patients suffered permanent harm or death due to a medication error (Valentin et al., 2009).

Herout \& Erstad (2004) conducted an observational study in a 16-bed surgical ICU and illustrated problems in administration of continuous infusions related to weightbased infusions. The error rate was reported per 1,000 patient days (105.9). The study was short in duration and did not observe any severe error consequences, however, $87.6 \%$ of weight-based infusions were incorrect due to relying on weight "estimates" or unreliable admission weights leading to erroneous doses being administered (Herout \& Erstad, 2004).

Another study looked at medical errors and adverse events in two ICUs and found a rate of 80.5 AEs per 1,000 patient days. Twenty-five of the 79 patients included in study sample suffered multiple AEs. Of the 120 AEs that occurred, $45 \%$ were preventable, meaning an error took place. Medications were involved in $47 \%$ (56) of adverse events, 19 of which were determined to be preventable ADEs, meaning a medication error was involved. Of serious errors reported, $78 \%$ were attributable to medication administration errors. Primarily, wrong dosage errors attributed to failure to carry out treatment correctly (Rothschild et al., 2005). Rothschild and colleagues used the most comprehensive method for detection of errors, using mixed methods of 
observations and retrospective chart reviews, as compared to similar studies during this time period but was still limited in generalizability to all critical care. All critical care units are not created equal even in similar organizations such as teaching hospitals. Patient population/types, (e.g., surgical vs medical), severity of illness in population served, differing staffing models and institutional culture play a role in patient safety studies (Rothschild et al., 2005).

Most recently, due to the wide variability in data and methods to evaluate incidence, cost, cause and impact of medication errors, an integrative review specifically involving critical care MEs was conducted in 2016. Forty studies were included in this review and support the general consensus that data is not able to be systematically synthesized due to the heterogeneity in study methods, variables, definitions of errors and reporting systems as well as care settings (MacFie, Baudouin, \& Messer, 2016).

\section{Critical Care Nurse's Role in Medication Safety}

The medication process is complex and systematic which leaves a large margin for error and is considered one of the most potentially hazardous nursing tasks is the hospital setting (Evans, Berry \& DeWit, 2006). Hughes \& Blegen (2008) describe five stages of the medication use process as ordering/prescribing, transcribing, dispensing, administering and monitoring. Medication errors (ME) can occur at any stage of the medication management process and research suggests that the administration stage is where the highest risk for error exists (Aspden et al., 2007). As reported in a national study, $44 \%$ of harmful medication errors reported originated in the administration phase (Latif et al., 2013). 
Medication administration errors (MAEs) often become synonymous with "nursing' errors because nurses stand at what Reason (1990) termed the "sharp end" of medical errors as frontline caregivers. Often times, nurses are the sole administrators of medications and therapies in critical care as well as other settings. The intravenous route is commonly used in the critical care setting due to the nature of patients' fragile physiological state. The critical care nurse (ICU RN) is considered to be the provider responsible for monitoring for effects and reactions to medications and therapies. Critical care nurses have also historically been held responsible for verifying or "double checking" every order and every medication prior to administration. The bedside is where nurses spend a greater portion of their time with $26.9 \%$ of it dedicated to medicationrelated activities (Keohane et al., 2008). Nurses are trained in the five rights of medication administration: right patient, right medication, right dose, right route and the right time (Cohen, 2007, Chapter 4).

Management of intravenous medications. The majority of intravenous (IV) medications used in critical care require expertise in management and close observation by critical care nurses in the ICU setting and are listed as high-alert medications by the ISMP (Kane-Gill, Kirisci, Verrico, \& Rothschild, 2012). Medications typically used in critical care as IV or continuous infusions include vasopressors and catecholamines, insulin, anticoagulants, electrolytes, antimicrobials, sedatives and analgesics amongst others (Valentin et al., 2009). One recent project by O’Byrne, Kozub, \&Fields (2016) was aimed at reducing IV MAEs in a small (350-bed) community hospital's ICU in Southern California. The 2-phase project reported a 53\% reduction in errors using mixed educational methods. 
Intravenous (IV) medications and infusions have been found to have high incidences of error relating to administration techniques across care settings and error reporting databases. (Herout \& Erstad, 2004; Hicks \& Becker, 2006). IV medications were found to have higher incidences of errors and $22.9 \%$ of IV errors were identified as administration errors. In addition, $48 \%$ of MAEs were attributed to performance deficit and $28 \%$ to violation of procedures and protocols (Hicks \& Becker, 2006). A systematic review of MAEs in US hospitals reported that few studies addressed causes of IV MAEs (Keers et al., 2013). Two studies have found that errors involving IV intermittent (bolus) or continuous infusions have been found to cause more serious harm than other medication administration errors (Keers, Cook \& Ashcroft, 2013; Ohashi et al., 2014).

Technology, nursing and medication safety. The systems approach to medical and medication errors looks at the entire system and how to build one that helps nurses catch errors before they reach the patient as described by Reason $(1990,2001)$ and Donaldson (2007). A great deal of effort has been put forth to alleviate the burden of responsibility regarding medication safety from the nursing profession. Moving from individual responsibility to a system's responsibility helps to protect both patients and providers. The goal is to prevent nurses and clinicians from relying on their own memory and manual calculations for dosage and administration of medications. A 2013 survey by the American Society of Health-System Pharmacists (ASHP), reported an increased effort by hospitals and health organizations to support medication administration by integrating informatics, health information technology (HIT) and nursing documentation (Fox et al., 2015). Barcode technology and nursing clinical documentation via the eMAR have mitigated a lot of the errors in administering medications to the wrong patient, 
wrong time or wrong dose. Poon et al. (2010) reported a decrease in ADEs from $11.5 \%$ to $6.8 \%$ with the use of barcode assisted medication administration (BCMA) and eMAR as well as a decrease in potential ADEs from $3.1 \%$ to $1.6 \%$.

Currently, the medication administration process involves first, the bedside nurse scanning the patient's arm band and the eMAR (now a function within the EHR capabilities) verifies that it is the correct patient. The second step is scanning the medication's barcode to verify that it is the correct medication, correct dose and correct route. Finally, real time documentation in the eMAR verifies that medications are given at the correct time.

As with any new innovation, there is a period of transition and adjustment. Some studies have studied medication errors attributed to HIT systems errors as well as medical devices like infusion pumps. Studies found the issue to be multifactorial and reinforced that no one piece of equipment or technology will completely eliminate all medication administration errors (Husch et al., 2005). Themes that emerged from literature on causes of error attributed to technology/devices include noncompliance, workarounds, overrides and bypassing the pump's drug libraries (Giuliano, 2018; Halbesleben et al., 2010).

Medication administration was found to be the most common cause of medication errors reported to a national anonymous database for medication error reporting in 2002 (Hicks, Cousins \& Williams, 2004). Observational studies report variable causes of medication administration errors (MAE) as well as rates of MAEs as a percentage of overall medication errors (Calabrese et al., 2001; Kopp, Erstad, Allen, Theodorou, \& Priestly, 2006; Rothschild et al., 2005b). One such study of admissions at a Veterans 
Administration hospital found 27\% of study ADEs were related to medication errors and $13 \%$ of those were MAEs (Nebeker, Hoffman, Weir, Bennett \& Hurdle, 2005).

Studies substantiating the efficacy of all the integrated systems CPOE, BCMA, eMAR and the EHR on the reduction of ME and MAE are limited. In addition to the lack of reliable published studies using US data, those that are available are also limited by site/setting and interventions with and without use of HIT. A controlled trial study in 2002 found no impact on serious medication errors using smart infusion pumps in cardiac surgery patients (Rothschild et al., 2005a). Another study by ASHP in 2009 found 58\% reduction of overall medication errors while excluding wrong time errors, however this prospective study found no reduction in medication errors in the ICU setting and looked at only BCMA as an intervention (Helmons, Wargel, \& Daniels, 2009). These observational studies in the early 2000s were in the midst of introducing new HIT and informatics systems. They also relied mostly on voluntary reporting of ADEs, MEs and MAEs as well as sentinel events. This area needs more investigation to asses medication errors in the era of well-integrated technology and its effect on the incidence of these.

\section{Reporting of Medication Errors}

Experts have urged the need for a national database for mandatory reporting in order to track trends across the nation since the late 1990's (Kohn, Corrigan \& Donaldson, 1999). The IOM also called for development of new methods for measuring patient safety in the early 2000 's. in response to the data (IOM, 2004). Congress responded to these recommendations with the passage of the Patient Safety and Quality Improvement Act (PSQI) of 2005. The law called for the creation of a national network of databases by the Agency for Healthcare Research and Quality (AHRQ). It authorized 
the creation of Patient Safety Organizations (PSOs) thereby acknowledging the need and reliance on patient safety event data to make healthcare safer for patients, and also, that efforts to improve quality and prevent harm are hampered by under-reporting of AEs (AHRQ, 2008). PSOs are entities that engage in activities that address causes, interventions and risks to quality and safety of patient care. (AHRQ, 2013).

The Institute for Safe Medication Practices (ISMP) is an independent, non-profit organization and its national Medication Errors Reporting Program (MERP), accepts reports directly from practitioners (physicians, nurses and pharmacists) and consumers. ISMP MERP however, is not entirely anonymous, the database collects reporter's information and shares information about incidents with federal agencies and other practitioners but does not disclose identifying information of the reporter (ISMP, 2019). ISMP is included in AHRQ's federal list of Patient Safety Organizations (PSOs), and providers working with ISMP are able to obtain the confidentiality and protections provided under the 2005 law (AHRQ, 2008).

The FDA's safety information and adverse event reporting program, MedWatch, is a government regulated reporting database that is mostly voluntary but does have some mandatory requirements regarding serious $\mathrm{ADE}$ and sentinel events. Voluntary reports are accepted from healthcare professionals as well as consumers. Mandatory reports come from facilities, distributors, importers and manufacturers. (Rose, 2016)

MEDMARX is a national database originally developed by the United States Pharmacopeia (USP) in the late 1990's and in 2008, it was transferred to Quantros, a private commercial software company. The reports are internet accessible, anonymous but only accessible by organizations who pay for their software packages. It collects and 
tracks adverse drug events as well as medication errors in its national database which claims to be the largest in the United States with over 1.3 million medication error reports and over 40,000 adverse drug reaction reports. Reports are also voluntary and accessible only to subscribed entities (Quantros, 2009; Latif, et al., 2013).

Medication error rates. The literature shows a wide variability in medication error rates. The variability is due to overall underreporting, lack of a standard definition for medication errors across health care settings, and differences in the detection of medication errors tied to ADEs (Rothschild et al., 2005; Kiekkas, Karga, Limonidou, Aretha, \& Karanikolas, 2011). Voluntary reporting, chart reviews and computerized monitoring are three methods used by healthcare organizations in the United States to monitor drug safety issues, errors and ADEs (Ghandi, Seger \& Bates, 2000; Flynn, et al., 2002).

Reporting to national databases is voluntary, although highly encouraged by national professional and accrediting agencies. In a study that reviewed reports submitted to the MEDMARX database, there was an increase in reports as well as a rise in institutions reporting errors (Hicks, Cousins \& Williams, 2004). In 1999, the database recorded 6,224 reports and by 2002, there were 192,477 reports from 482 institutions throughout the United States. The increase demonstrates an interest by organizations in medication error reporting as well as a commitment to quality and safety of medication management (Hicks, Cousins \& Williams, 2004).

Underreporting. Though the general body of knowledge supports evidence that medication errors occur and may result in patient harm, under-reporting of events including near miss events has continued to plague the healthcare system. Results of a 
national survey of 1,652 hospitals in 2006 revealed that at the time, most hospitals had a centralized adverse event reporting system but only $32 \%$ had environments that supported reporting and only $13 \%$ had broad staff involvement in reporting. (Farley et al., 2008).

Studies have attempted to investigate error reporting rates along with actual medical and medication error rates as well as the reasons for underreporting practices amongst healthcare workers. Comparing observed safety events or errors with actual reported events is the most effective method to determine the extent of underreporting. This method is time consuming and not cost effective for organizations to realize. A systematic review of under-reporting of adverse drug reactions (ADRs) reported rates of 95\% underreporting for hospital-based studies which used various surveillance methods and spanned twelve countries (Flynn et al., 2002; Hazell \& Shakir, 2006). Levinson, 2010 suggest vast underreporting based on their findings of adverse events when compared to actual reports during the same time period that their data collection took place and concluded that only one in seven errors are actually reported.

An Australian comparative study of two major academic hospitals also found very low rates of filed incident reports as compared to medication errors identified at audit. Only 1.2 per 1000 of prescribing errors has accompanying incident reports. Medication administration errors were detected in $27.4 \%$ of all medication administrations but none had an incident report filed (Westbrook et al., 2015).

Research has demonstrated that culture in a healthcare setting is the most important contributor to safety (TJC, 2018). Creating a culture of safety involves transparency, non-punitive practices, spontaneous incident reporting including self- 
reporting of not only sentinel events or adverse events but also near misses (Kohn, Corrigan \& Donaldson, 1999). The Joint Commission issued a sentinel event alert in 2018 calling and urging healthcare organizations to begin laying the groundwork for creating a reporting culture. It acknowledges that reporting provides crucial information about system failures. It is imperative to be able to understand what factors influence reporting as well those that act as deterrents to event reporting (TJC, 2018). Only with this knowledge can a healthcare system truly become safer and provide safe and efficient care without harm.

Despite the recommendations and guidelines from various expert agencies, reporting of medical errors, including medication errors, continues mostly voluntary and non-standard across the nation. It should be noted that currently, "each accredited organization is strongly encouraged, but not required, to report sentinel events to The Joint Commission" (TJC, 2019, "Sentinel Events Policy and Procedures," para.3). It is generally assumed that the number of actual patient safety events, including medical errors and medication errors that do not result in serious patient harm (near-miss events), is widely underreported.

\section{Barriers to Error Reporting}

According to the literature, the process of error reporting is systematic. It involves recognizing that an error has taken place, assessing the need to report an error, preparing and incident report, and follow-up response from leadership or those who receive the report (Wakefield, B.J., Uden-Holman, \& Wakefield, 2005).

Using an anonymous survey/questionnaire method to study nurses and physicians at acute care hospitals, three studies had similar designs looking at barriers to error 
reporting. Each study was carried out in different nations with similar barriers to event reporting, suggesting the issue of underreporting is a global phenomenon (Evans et al., 2006; Stergiopoulos et al., 2016; Uribe et al., 2002). Their findings classified barriers to event/error reporting into three categories; organizational, educational and individual or cultural (Evans, Berry, \& DeWit, 2006; Stergiopoulos, Brown, Felix, Grampp, \& Getz, 2016; Uribe, Schweikhart, Pathak, \& Marsh, 2002; Wakefield, B.J., Uden-Holman, \& Wakefield, 2005).

Organizational barriers included time to document event, extra time/work involved in reporting, length of forms, anonymity of report, and paper forms or computers not available. Educational barriers include the belief that a good outcome did not warrant a report, little value in reporting to improve quality, lack of feedback, lack of knowledge in usefulness of reporting, not knowing how report and incident, and being unaware of who is responsible for filing a report. Cultural barriers include belief that reporting is accusatory or "telling" on someone and fears of litigation, blame or shaming, discipline or punishment and/or termination. Individual barriers include a lack of interest in error prevention and lack of interest in reporting. Two of the studies strictly looked at nurses and physicians, but did not include barriers involving other professions involved in medication management, like clinical pharmacists (Evans, Berry, \& DeWit, 2006; Stergiopoulos, Brown, Felix, Grampp, \& Getz, 2016; Uribe, Schweikhart, Pathak, \& Marsh, 2002; Wakefield, B.J., Uden-Holman, \& Wakefield, 2005).

One Canadian qualitative study used focus groups to explore medication error reporting in four community hospitals. The study found gaps in communication and education amongst physicians, nurses and pharmacists (Hartnell, MacKinnon, Sketris, \& 
Fleming, 2012). Also included in this research, were interviews with leadership from each hospital, risk managers, which revealed discrepancies in communication between leadership and hospital staff regarding education on errors, reporting and feedback. Results of the study revealed five major barriers to reporting as; reporter burden, professional identity, information/knowledge gap, organizational factors and fear (Hartnell et al., 2012). Both Sujan (2015) and Stergiopoulos et al. (2016) found education to be one of the major barriers to error reporting. In the first study, semi structured interviews revealed a general confusion amongst front line staff regarding the incident reporting process (Sujan, 2015). The latter, utilized a survey method to investigate all healthcare providers including pharmacists, nurses and physicians. It was reported that $52 \%$ of survey respondents were uncertain about the reporting procedures Stergiopoulos et al., 2016).

Nurses and error reporting. Nurses have the greatest opportunity and potential to impact on the safety of the environment and the system in which they work. Nurses have been found to be the main users of event reporting systems. Milch et al. (2005) reported registered nurses provide nearly half of the total incident reports reviewed from 26 acute care hospitals. In their study, Evans et al. (2006) reported $88.3 \%$ of nurses would know how to access an incident report form, $89.2 \%$ had ever completed a report and $81.9 \%$ would know what to do with the completed report. Another study using internal reports at two U.S. hospitals found $88 \%$ of reports were filed by nurses (Nuckols, Bell, Liu, Paddock, \& Hilborne, 2007).

Studies list variable rates of involvement in reporting by different professions depending on the study variable. Milch et al., (2005) looked at general error and adverse 
event reports as did Nuckols et al., (2007). Stergiopoulos et al. (2016) used a survey to look at ADEs and had $27 \%$ of respondents identifying as nurses compared to $15 \%$ for physicians and more than $40 \%$ by pharmacists.

Knowledge of error reporting process. Despite nurses' familiarity with event reporting systems, the types of events being reported most often include falls, deep vein thrombosis and pressure injuries (Evans, et al., 2006). Three studies reported a discrepancy in the percentage of medication related adverse events that occur when compared to reported ADEs and rates of reported near miss events varied between $4.4 \%$ 13\% of all reports (Milch et al., 2005; Nuckols et al., 2007; Stergiopoulos et al., 2016). According to Evans et al., (2006) 97\% of nurses were reported to regard drug error "near misses" as the least important incident to report.

Ulanimo, O’Leary-Kelly and Connolly (2007) found respondent nurses in their study cited lack of knowledge on policies and procedures as the major contributor to not reporting their errors. The results suggested to researchers that there is a gap in the nurses' perceived knowledge regarding medication errors and reporting practices. (Ulanimo, O’Leary-Kelly, \& Connolly, 2007). Sanghera, Franklin and Dillon (2007) conducted a mixed methods study in the UK to assess ICU staff on medication errors and reporting practices. Where this study was not specific to nurses, the themes identified, demonstrated no significant difference in attitudes toward errors and reporting in the ICU as compared to US studies in other settings.

Some more recent studies have looked at all hospital nurses and found a lack of knowledge of the medication error reporting process as well as lack of information/ feedback regarding filed reports contributed to underreporting (Joolaee et al., 2011; 
Rutledge, Retrosi, \& Ostrowski, 2018). Dirik., Samur, Seren Intepeler, and Hewison (2019) reported $66 \%$ of respondent nurses did not recognize an error had been made, $63.5 \%$ believed reporting to be unnecessary if there was no patient harm, and $54.2 \%$ considered an error not serious enough to report. As far education and training, $37.5 \%$ respondents reported lack of training about medication errors, 39.6\% reported the organization lacked a clear definition of medication error and $29.2 \%$ were unaware of the existence of an error reporting form.

A review of the literature suggests that medical errors are hard to quantify empirically but are common in the hospital setting despite decades of efforts from governmental and private organizations. Medication errors are broad and are a significant cause of morbidity and mortality in US hospitals. Medication error rate estimates are variable, and the evidence is just as heterogenous as the methods used to detect medication errors of all types. Critical care units are perhaps where patients are most vulnerable and susceptible to medical and medication errors/adverse events. Critical care nurses are the last line of defense for the ICU patient against harm from medical errors and can provide valuable information regarding safety concerns as frontline staff. The literature suggests that this information is widely underreported. Event reporting provides valuable data that can be used to address latent system problems affecting patient safety before they reach the patient and eliminate or at least minimize harm. A crucial concept of safety culture is creating a reporting culture that encourages and/or rewards incident/ safety reporting of medication/medical errors and near miss events. The literature suggests that one major contributing factor to underreporting is a general lack of knowledge and education regarding medication error reporting processes, policies, 
definitions and expectations. In a setting such as critical care, it is imperative to have nurses whom are empowered with knowledge and education to report errors and near miss events in order to improve care quality, health outcomes and prevent harm. 


\section{Theoretical Framework}

Ajzen and Fishbein's Theory of Reasoned Action (TRA) in conjunction with its modified version; Theory of Planned Behavior (TPB) (Ajzen, 1991; Ajzen \& Fishbein, 1980; Fishbein \& Ajzen,2005), will be utilized as the theoretical framework for this project. The TRA illustrates relationships among beliefs, attitudes, intentions and certain behaviors. It used the concept of intentions based on known information about a behavior (Ajzen, 1991). The TRA functions under the assumption that people will make decisions and form intentions to perform a certain behavior based on what they know about the behavior (Kuhns \& McEwen, 2011).

In terms of this project 's objective, the constructs developed by Ajzen \& Fishbein (1980) allowed exploration of variables that form attitudes toward error reporting and form a nurse's intention to report medication administration errors. The TPB builds upon the TRA to further describe variables that influence an individual to either to act or not on their intentions based on their perceptions of behavioral control. (Montano \& Kasprzyk, 2002).

Following the TRA and TPB models, behavioral beliefs, normative beliefs and control beliefs are variables influencing an individual's attitudes, subjective norms and perceived behavioral control toward reporting medication errors (Ajzen \& Fishbein, 1980). Based on the assumptions of the TRA and TPB, it is assumed that favorable attitudes toward a given behavior, such as medication error reporting, make it more likely that an individual will form the intention and ultimately follow through on performing the desired behavior (Ajzen, 1991). Behavioral intention is a concept based on this theoretical model. Intention is formed by factors influencing three categorical beliefs 
which in turn, influence attitudes, perceived norms and perceived behavioral controls toward a certain behavior (Montano \& Kasprzyk, 2002).

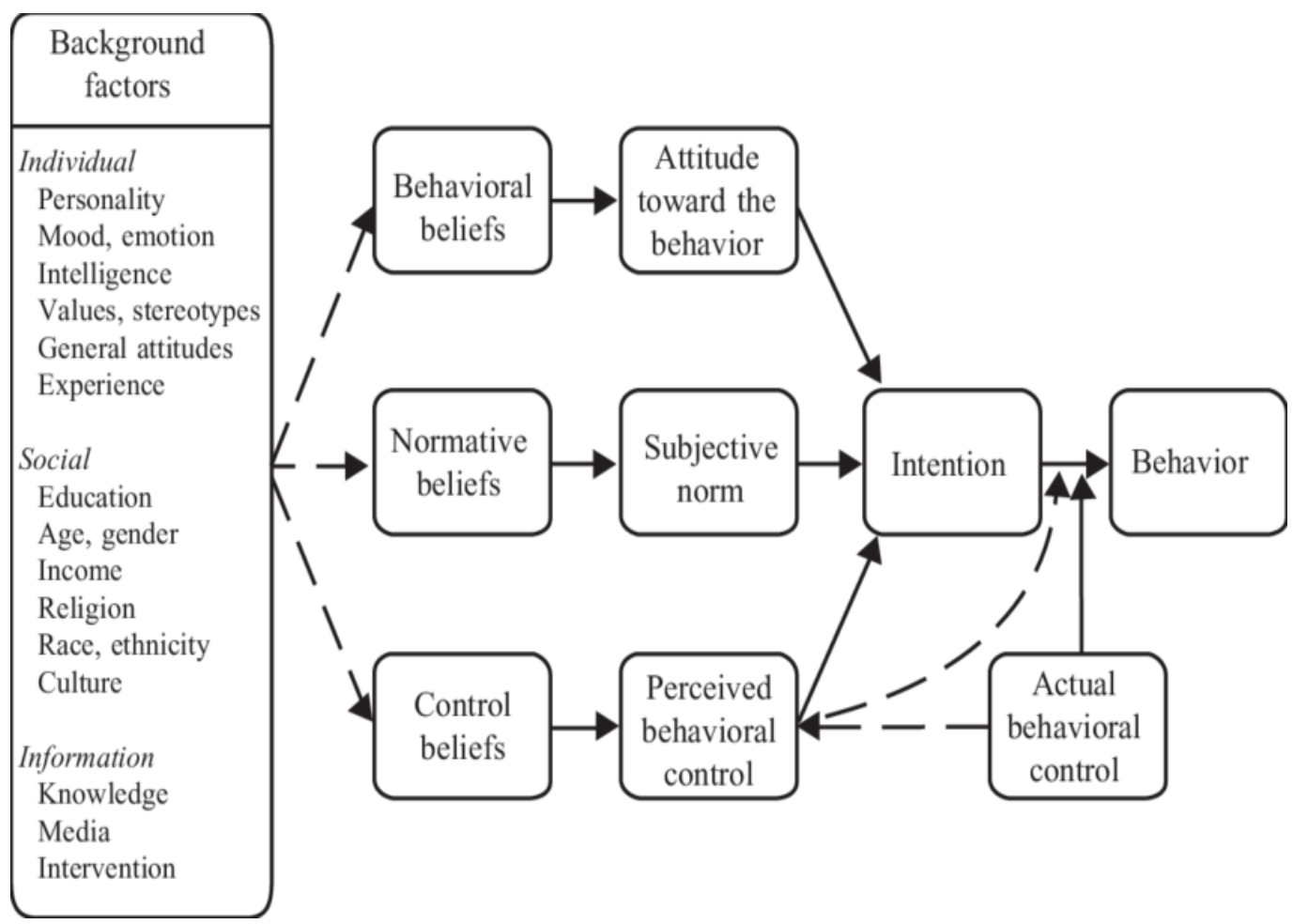

Figure1. The theories of reasoned action and planned behavior. Adapted from "The influence of attitudes on behavior," by I. Ajzen, and M. Fishbein, 2005, Handbook of attitudes and attitude change: Basic principles (p.194). Copyright 2005 by I. Ajzen \& M. Fishbein.

Behavioral beliefs translate to a nurse's perceived positive or negative consequences of reporting error and can influence their attitudes toward medication error reporting. For example, if a nurse had only seen negative consequences form reporting such as disciplinary action, lack of action/change or does not receive positive feedback from leadership, this would create negative attitudes toward reporting. In turn, this would negatively influence behavioral intention and a nurse would not form the intention to report a medication error when one is encountered (Russo et al., 2015). One study found that gaps in communication between leadership and nurses can lead to formation of 
negative behavioral beliefs about error reporting. Poor communication prevents feedback from reaching the right people, creating negative attitudes toward error reporting and making it less likely that medication errors will be reported. (Hartnell, et al., 2012, p. 58).

Normative beliefs pertain to the culture that surrounds reporting of medical and medication errors. Nurses' perceived social pressure toward reporting medication errors is dependent on whom they consider to be part of their culture whether it be only bedside nurses, physicians, pharmacists, administrative RNs/ clinical managers or all health professionals. This variable is dependent on who the nurse considers important to them within their setting/ organization. Creating a partnership amongst healthcare professionals and leadership is crucial when considering a cultural change. For example, the approval or disapproval of reporting by peers, colleagues and/or superiors would influence whether a nurse will submit to perceived social norms. Making medication error reporting an expected behavior or "social norm," would positively impact normative beliefs, create positive subjective norms regarding reporting errors and make it easier to form the intention to report an error (Russo et al., 2015).

Control beliefs refer to the system factors that facilitate or impede the formation of a behavioral intention to report errors. The belief that one has the control over one's behavior is crucial to formatting an intention. Perceived behavioral control describes nurses' perceptions of the ease or difficulty of reporting medication errors. This concept can have a direct effect on nurses' intentions to report medication errors (Russo et al., 2015). 
It was inferred from these concepts that providing nurses with education and knowledge regarding the positive outcomes from error reporting can help shape behavioral beliefs and create positive attitudes. Providing real time and updated knowledge regarding safety /just culture, systems failures or near misses and what is expected behavior can help shape normative beliefs and create subjective norms that supports the intention to report a medication error. Safety and just culture can help ensure nurses feel comfortable and reassured that they are supported in reporting medication errors. Finally, providing continuous information, education and knowledge regarding reporting avenues, policies and processes for reporting can influence the development of control beliefs and nurses' perceived behavioral control. A nurse with sufficient training and knowledge may form the intention to report an error and will do so if they believe that they can easily act on their intention.

This project hypothesized that certain variables help to facilitate or hinder the formation of a nurse's intention to report a medication error. Modifiable background factors include education and knowledge which can influence a nurse's behavioral, normative, and control beliefs (Montano \& Kasprzyk, 2002). A study of 188 Italian nurses used a similar approach using this theoretical framework for exploring motivations that influence reporting behaviors (Russo, Buonocore, \& Ferrara, 2015). These concepts provided guidance for this project which sought to explore the educational and knowledge factors that affect behavioral, normative and control beliefs in critical care nurses about medication error reporting. 


\section{Method}

\section{Purpose}

The purpose of this project was to explore the knowledge base of critical care nurses in relation to medication error reporting. This information was then used for identifying knowledge gaps and any needed enhanced education.

\section{Design}

This exploratory project utilized a one-time, self-reported survey design (Appendix A). Data was collected using a 10-question survey developed by the student researcher. The survey was developed and guided by themes identified in the literature regarding medication error underreporting and the need for understanding the definition of medical errors, what constitutes medical errors and the documentation process. The survey was reviewed by two quality control managers (QCMs) and two senior MSN student classmates for readability. Both QCMs and RNs stated they felt comfortable completing the survey, understood the intent and were not confused by the wording in any of the questions. No data was be collected on years of service, education, gender, age or any other identifying data. No RN or patient demographics or data were obtained. Gaps in knowledge were identified from data analysis and results guided recommendation for enhanced educational development in this content area.

\section{Sample and Site}

The project took place at a large 750-bed academic acute care hospital. A convenience sample was used that included all inpatient nurses who work in the ten critical care units, including step down units. These units are all housed within the same hospital and were surveyed over the same timeframe of two weeks of beginning March 9, 2020 and ending March 23, 2020. Units varied in size from 8-22 beds and vary in staffing 
ratios between units and shifts. No critical care units were excluded from the project sample. RNs included in this sample were staff nurses both full time and part time as well as per diem RNs and those designated as critical care float RNs whom float only within the critical care units. Nurses not currently on active duty, travel RNs and those new graduate RNs currently on orientation were excluded from the project sample as well as those that were on leaves of absence or vacations as they were not able to participate.

\section{Procedures}

The Chief Nursing Officer (CNO), the Quality Control Manager (QCM) for critical care as well as the Critical Care Director of Nursing Services, Assistant Clinical Managers (ACM) and Clinical managers (CM) of individual critical care units have been informed of this project and have given written support in October 2019 via electronic communications (Appendix B). This project was submitted for IRB review at the subject facility as well as Rhode Island College's IRB in January 2020 and received exempt research status by both review boards in February2020.

After completing IRB review,, an information email was disseminated with the help of the CMs/ACMs of the units to prepare the staff RNs for the upcoming survey. The email provided information on the purpose of the project and timeframe in February 2020 (Appendix C). The student researcher also attended several staff morning huddles on critical care unit as allowed by $\mathrm{CM}$ to introduce the project and answer questions (Appendix D). A flyer designed by the student researcher and approved by the study facility and Rhode Island College's IRB was displayed on each units' staff lounge bulletin boards (Appendix E) to aid in recruiting participants for the survery. Paper copies of the survey with an informational letter attached (Appendix F) were distributed to all 
critical care units on Monday, March 9, 2020. They were placed in a central location recommended by the units' clinical leadership and a sealed box for completed surveys was provided on each of the units. Completed surveys were kept in a locked desk drawer in a locked office and were shredded upon completion of the project.

RNs were allowed two weeks to complete the survey. Reminder emails were sent after completion of first week on March 16, 2020 by the student researcher to CM/ ACMs to distribute to staff lists. Also, a visit to the units was made on each shift (day, eve, night) by the student researcher after first week to give opportunity for questions regarding the project and encourage participation.

\section{Measurement and Analysis}

Data collection ended on Monday, March 22, 2020. The survey had a total of 10 questions regarding knowledge on the definition of medication errors, detection of medication errors, reasons for underreporting, and the documentation process. The survey had a mixed format of questions: five yes or no, four true or false and one question which required respondents to recognize a medication error when posed with a short case-study type scenario (yes or no). All questions required the respondent to circle their response. Total time to complete the survey was deemed to be less than five minutes. Questions were analyzed using descriptive statistics. Analysis was performed to determine the degree to which the aggregated results demonstrated a knowledge of the definition of medication errors, recognition as well as knowledge of hospital policies and processes regarding medication error reporting. Gaps identified through data analysis were reported to the PI and shared with nursing leadership along with recommendations for possible education programs that would help close those knowledge gaps. 


\section{Ethical Considerations}

While there are no patients directly involved in this project, ethical considerations were directed toward the nurses who provide responses to the project survey.

Participation was completely voluntary. Consent was assumed if survey information was completed. The student researcher was a current employee of the subject facility where data was collected which could pose undue pressure to nurses to participate. It is highly unlikely that identifying information could have been shared inadvertently. In the rare event that this occurred, the student researcher mitigated this by maintaining privacy and did not disclose any information obtained in the data collecting phase of this project. There was also possibility that colleagues will feel pressured to complete the survey and emit responses since they have developed personal relationships and a sense of camaraderie with the student researcher over their years of service. The student researcher maintained ethical integrity and confidentiality by not discussing the project during work hours and maintained objectivity by not sharing an opinion or feelings on the project with any colleagues that comprised the project sample.

\section{Evaluation and Plan for Dissemination}

This project aimed for a $30 \%$ participation rate and was evaluated by assessing if it revealed nurses' knowledge to medication error reporting could be addressed in future educational projects. At the end of the given timeframe for this project, a response rate of $25.66 \%$ was achieved with 77 completed surveys collected.

Dissemination took place via a major project presentation at the Rhode Island College Nursing Education Center (RINEC) on May $5^{\text {th }}, 2020$. A brief report was provided to the newly appointed Interim chief nursing officer $(\mathrm{CNO})$, quality control 
manager (QCM) and the director of nursing in critical care to distribute at their discretion to all critical care unit leadership. The student researcher also provided results via a onepage report to be displayed on the units' quality and safety board at the discretion of nursing leadership. This major project was also be uploaded to digital commons on RIC's website in the Sumer of 2020. 


\section{Results}

The survey was made available for two weeks from Monday March 9, 2020 to Monday March 23, 2020. The critical care division director reported that there were approximately 350 critical care nurses currently on staff whom were eligible to participate according to staff lists. After allowing for medical/personal leaves, those on administrative leaves and employees who were on approved vacations during the project timeframe, the total number of eligible nurses was roughly 300. Each unit was provided with 30 blank surveys with an informational letter attached. After the first week, 31 completed surveys were collected. After the second week, an additional 46 surveys were collected for a total of 77 surveys collected within the given project timeframe $(N=77)$. Total respondents amounted to a $25.66 \%$ response rate for this project. It was not possible to make the survey available for any additional time or to increase recruitment methods due to a national public health crisis affecting normal hospital operations. Each of the ten survey questions was in either yes/no or true/false format. Each question was analyzed individually using descriptive statistics and then grouped into categories of knowledge of medication error policies, knowledge and beliefs of reporting procedures and recognition of medication errors. The responses were analyzed using categories in order to draw conclusions for recommending future education programs aimed at critical care nurses.

Question one asked nurses whether they were aware of the hospital's definition of medication error. The majority, $60.53 \%$, of respondent nurses indicated that they were not aware of any formal adopted definition while $39.47 \%$ indicated they had this knowledge. Table one displays percentages of all respondent nurses that were aware of the adopted definition. 
Table 1

Are you aware of hospital's definition of medication error? $(N=77)$

\begin{tabular}{cc}
\hline & $N(\%)$ \\
Yes & $39.47 \%$ \\
No & $60.53 \%$
\end{tabular}

Question two was a two-part question inquiring about the nurses' ability to locate hospital policy regarding medication errors and whether or not they have read it. Table 2 displays responses to question two, part (a). There was one respondent who indicated "yes" to knowing how to find the hospital's policy on medication errors but did not answer the second part of the question indicating whether or not they had read the policy. Overwhelmingly, $76.62 \%$ of respondents indicated knowing where to find the policy while $23.38 \%$ of all respondent nurses answered that they did not know how to find the hospital's policy. The second part of question 2 (b) follows up by asking whether the nurses that responded "yes," to the first part had actually read the hospital's policy. This data is displayed in Table 3.

Table 2

Do you know how to find the hospital's policy on medication errors? $(N=77)$ 
Table 3

\begin{tabular}{rr}
\hline If yes, have you read the hospital's policy? $(n=59)$ & \\
\hline & $n(\%)$ \\
Yes & $28.81 \%$ \\
No & $71.19 \%$ \\
\hline
\end{tabular}

This data demonstrates that having knowledge on a process does not mean nurses will be able to use or follow through on this knowledge. Of those that responded "yes," to question 2(a), 69.49\% $(n=59)$ had not read the hospital's policy on medication errors despite reporting that they know where to find it. A small percentage of nurses have followed through with reading the policy. This equates to $22.37 \%$ of respondent nurses whom have both the ability to find the hospital's policy and have also read it. This leaves $77.63 \%(n=76)$ of respondents that do not have knowledge of the hospital policy on medication errors because they either do not know how to find it or know where to find it but have not read it. Of note, the written policy available online by the subject facility where the survey took place, includes the adopted definition of a medication error as adopted rom the National Coordinating Council for Medication Error Reporting and Prevention (NCC MERP). It specifically addressed the definitions of "actual medication error," "prevented medication error" or near miss error, and "potential medication error." Question three asked nurses to differentiate between a near miss event and a medication error. Of note, as described above, the subject facility, per their written policy, considers a near miss event/error ("potential medication error") as a medication error. It is defined in this policy as "one that was stopped before reaching a patient" (Rhode Island Hospital, Quality and Error Management Policy PH-566, 2019). A 
medication error and a near miss event were indicated to be the same by $30.26 \%$ of respondent nurses whom circled "true" to this statement on the surveys. On the contrary those that circled "false" represented $69.74 \%$, a majority, inferring that they believe a near miss event is not a medication error. Table 4 displays respondent results as percentages.

Table 4

\begin{tabular}{cr} 
A near miss event and a medication error are the same. $(N=77)$ \\
\hline \\
True & $30.26 \%$ \\
False & $69.74 \%$ \\
\hline
\end{tabular}

Question four asked nurses to judge whether a medication error should be reported based its effect or harm to the patient. Only $2.60 \%$ of nurses responded "true" to the statement that an event that does not cause harm, does not need to be reported. Table 5 displays the results as percentages of overall respondents. This data demonstrates that $97.40 \%$ of nurses believe that incidents involving medications need to be reported even if they do not cause harm to the patient.

Table 5

If there is no harm to the patient, an incident involving a medication does $\underline{\text { not }}$ need to be reported. $(N=77)$

\begin{tabular}{cc}
\hline & $N(\%)$ \\
True & $2.60 \%$ \\
False & $97.40 \%$ \\
\hline
\end{tabular}


Question five asked whether the nurses have received any type of education and/or training regarding medication error prevention strategies. Table 6 displays the results as percentage of respondent nurses that report "yes" to receiving education and/or training. Those that responded "no," were equal to $28.57 \%$ of respondent nurses inferring they have not received education or training on medication prevention errors.

Table 6 Have you received education/training regarding medication error prevention strategies? $(N=77)$ $N(\%)$

Yes $71.43 \%$

No $28.57 \%$

Question six asked whether nurses knew how to access the hospital's event reporting system (Safety Net). Table 7 below displays this data in as a percentage of all respondent nurses. The majority, $93.51 \%$, or nurses reported having knowledge of how to access the online event reporting system.

Table 7

Do you know how to access the hospital's incident reporting system (Safety Net)? $(N=77)$

Yes

$93.51 \%$

No

$6.49 \%$

It is important to note that this is an umbrella electronic program that addresses safety issues or concerns system wide. Any employee with access to the facility's intranet is able to access it regardless of job title or credentials. Anything affecting safety, from 
patient falls, medical equipment failures, behavioral issues, medical/medication errors and technology issues, can be reported via this program and are then funneled to the appropriate leadership personnel to address the issue.

Question seven followed up in order to asses usability of the system as perceived by the population that utilizes it most, according to the literature. This question asked if nurses believe reporting a medication error is fast and easy and $75.32 \%$ nurse participants responded "no." Table 8 displays the data in $\%$ of total respondents $(n=59)$ who also hthe knowledge on had howto access the hospital's reporting system.

Table 8

Do you believe reporting a medication error is fast and easy? $(n=59)$ $n(\%)$

Yes $24.68 \%$

No $75.32 \%$

Question 8 asked nurses whether they believe reporting medication errors will result in disciplinary actions. Two respondents left this answer blank while $24.68 \%$ of nurses indicated this to statement by circling "true." Table 9 displays results of this data demonstrating that the majority of respondent nurses do not believe reporting a medication error will result in discipline. 
Table 9

Reporting a medication error will result in disciplinary action. $(n=75)$

\begin{tabular}{lc}
\hline & $n(\%)$ \\
True & $24.68 \%$ \\
False & $72.73 \%$ \\
Unanswered & $2.60 \%$ \\
\hline
\end{tabular}

Question 9 tested the nurses' knowledge of adverse events (ADEs) as related to medication errors and reporting by asking whether nurses believe all ADE's should be reported. Table 10 displays results in which, $93.51 \%$ of nurses believe all adverse drug events should be reported via the hospital's event reporting system. A small percentage of respondents, 6.49\% answered "no" indicating they do not believe all ADEs should be reported.

Table 10

All adverse drug events should be reported via the hospital's reporting system (Safety Net). $(N=77)$

\begin{tabular}{rr}
\hline & $N(\%)$ \\
Yes & $93.51 \%$ \\
No & $6.49 \%$ \\
\hline
\end{tabular}

Question 10 was meant to elicit the knowledge needed from nurses in identifying a medication administration error. A short scenario was introduced where a patient's weight was incorrectly entered into an infusion pump and lead to a discrepancy between the order in the electronic medical record and the infusion rate on the pump in milliliters per hour $(\mathrm{mL} / \mathrm{hr})$. In the scenario, there was no harm to the patient. Table 11 below 
demonstrates that a majority, $96.1 \%$, of respondents were able to correctly identify this to be a reportable incident.

\begin{tabular}{lr} 
Table 11 & \\
\hline After reading case scenario, is this a reportable incident? & $(N=77)$ \\
\hline & $N(\%)$ \\
Yes & $96.10 \%$ \\
No & $3.90 \%$ \\
\hline
\end{tabular}

To further analyze the data, three main themes were explored which arose from responses to the survey questions. Mainly, knowledge gaps were considered to be either hospital policy-related, reporting system-related or error recognition-related. Table 12 displays data related to hospital policy and definitions, table 13 displays data related to the reporting system and its usability, and table 14 displays data related to error recognition/identification. Only one question explored the theme of fear of disciplinary action amongst critical care nurses related to medication error reporting.

Questions one, two and three explored the general knowledge base of the critical care nurses related to the hospital definition and policies regarding medication errors. Overwhelmingly there was a knowledge gap identified with $60.53 \%$ of nurses not being aware of the facility's adopted definition of a medication error. A majority of nurses, $77.93 \%$, have not read the hospital's policy despite most respondents indicating that they know where to find it. Lastly, $69.74 \%$ of nurses lack the knowledge that near miss events are in fact medication errors as per the facility's written policy. Table 12 below displays summarized results in percentages related to hospital policy and procedures. 
Table 12

Nurses' Knowledge Regarding Definition and Policy on Medication Errors

$(N=77)$

$N(\%)$

Nurses do not know the hospital's definition of a medication error

$60.53 \%$

Nurses have not read or cannot find hospital's policy on medication errors

$77.93 \%$

Nurses cannot identify medication errors versus near miss events as both reportable

$69.74 \%$

Table 13 below summarizes the knowledge and beliefs explored in questions four, six, seven and nine. With regard to when and how to access the reporting system and its usability as perceived by respondent nurses, data demonstrates that nurses reported having the knowledge of what types of incidents need to be reported. Of all respondent nurses, a vast majority, $>90 \%$ of respondents, reported knowing all incidents involving medication errors should be reported regardless of harm, all ADE's need to be reported and reported knowing how to access the hospital's reporting system. Only $24.68 \%$ of respondents reported that they believe reporting medication errors is fast and easy.

Table 13

Nurses' Knowledge of When/How to Access Reporting System and Usability

$\frac{(N=77)}{N(\%)}$

Nurses believe all incidents involving medications need to be reported, $\quad 97.40 \%$ regardless of harm.

Nurses believe all adverse drug events should be reported $\quad 93.51 \%$

Nurses know how to access reporting system (Safety Net) $\quad 93.51 \%$

Nurses believe reporting medication errors is fast and easy $\quad 24.68 \%$ 
Questions one, three, five, and ten explored medication error definition, recognition and prevention. It is important for nurses to know what a medication error is, how to prevent one and sufficient education and knowledge to be able to recognize a medication error when one occurs. Overall, nurses had a high level of knowledge in a specific scenario (question \#10) with $96.10 \%$ being able to correctly identify a medication error. Also, $71.43 \%$ of nurses reported having received education on error prevention strategies. Table 14 summarizes the findings of these questions and demonstrates a gap in the knowledge when it comes to defining medication errors. Ranging between $30-40 \%$ of respondent nurses were aware of the definition but were unable to differentiate from a near miss events. Only $30.26 \%$ of nurses knew that near miss events are considered a type of medication errors that fail to reach the patient and are considered as reportable medication errors in the hospital policy. This is a significant finding because near miss events are crucial incidents that could reveal system wide issues that could prevent patient harm in the future.

\section{Table 14}

Nurses' knowledge of medication errors (definition, identification and prevention)

$(N=77)$
$N(\%)$

Nurses are able to correctly identify a medication error $96.10 \%$ Nurses received education on medication error prevention strategies $71.43 \%$

Nurses are aware of the hospital's definition of medication error $39.47 \%$ Nurses believe near miss event and medication error are the same $\quad 30.26 \%$ 


\section{Summary and Conclusions}

Medical errors, including medication errors, continue to be one of the major causes of injury and/or death in the United States (Makary \& Daniel, 2016). Patients hospitalized in critical care units are especially vulnerable to medical and medication errors (MacFie, Baudouin \& Messer, 2016). Providers, especially critical care nurses stand at the sharp end of medical errors which puts them in the most poignant position to be able to shed light on root causes of medication errors. Reporting of medication errors is a tool that can be used to establish systems processes to mitigate and prevent medication errors from reaching patients and causing harm. To be able to have a robust reporting culture that supports education and medication safety requires bedside nurses to be empowered to report all adverse drug events, near miss events and medication errors.

Assessing critical nurses' knowledge of medication error reporting practices as well as medication safety techniques in their practice would allow nursing leadership to gain insight into the resources and education needed to cultivate and improve the culture of safety and overall outcomes for hospitalized patients in critical care units. This information would also increase nurse competence and confidence in medication error reporting practices. Patient and nurse satisfaction can be improved if enhanced education programs aimed at critical care are considered and acted upon.

The purpose of this project was to explore the knowledge base of critical care nurses in relation to medication error reporting. This information would then be used for identifying knowledge gaps and needed enhanced education. This project was reviewed by the Lifespan and Rhode Island College Institutional Review Boards.

According to the theories by Ajzen and Fishbein, the concept of intention based on known information about a behavior (Ajzen, 1991) rests upon a subject's behavioral, 
normative and control beliefs and their influences over attitudes, subjective norms and perceived behavioral control (Ajzen \& Fishbein, 1980). Translated to this project's subject matter, essentially: a nurse's perception of the consequences of reporting errors, the reporting culture at subject facility and the perceived ease of reporting procedures can influence whether or not a nurse forms the intention to report a medication error. This project hypothesized that addressing possible knowledge gaps may serve to affect a nurse's beliefs (behavioral, normative and control) regarding medication error reporting and ultimately positively influence attitudes, subjective norms and perceived behavioral control toward medication error reporting causing more intentions and follow through with reporting behaviors.

To collect data for this project, a survey was formulated by the student researcher and distributed to a convenience sample of critical care nurses at a large acute academic hospital. A description of the one-time, voluntary survey was presented to potential participants during staff meetings/huddles, by email, a flyer and in an informational letter attached to surveys in the staff breakroom.

The survey consisted of 10 questions that would explore the knowledge base of the nurses regarding medication errors and reporting of these. Respondents answered each question with a yes/no or true/false format. The survey was available for two weeks. No demographic data was collected on the subjects to maintain anonymity and confidentiality. There were 300 potential participants, with a final response rate of $25.66 \%(N=77)$ by the end of the two-week timeframe allowed.

Among the 77 participant nurses, $60.53 \%$ were not aware of the facility's adopted definition of medication error, $71.19 \%$ of nurses responded they have not read the 
hospital's policy despite having knowledge of how to find it, and $69.74 \%$ were not aware that near miss events are medication errors. A significant percentage, $24.68 \%(n=75)$ of critical care nurses in this project sample, believe that reporting a medication error will result in disciplinary action. Respondent nurses demonstrated a high knowledge base regarding medication error reporting. Incidents involving medications that do not cause patient harm were deemed reportable by $97.40 \%$ of participants, $71.43 \%$ of respondents reported having received education on error prevention strategies and $96.10 \%$ of nurses correctly identified a reportable medication error.

Survey questions were analyzed in categories of knowledge deficits that were either hospital policy-related, reporting system-related or error recognition-related. The three categories overlap and play key roles in how critical care nurses are able to safely administer medications to a population with a highly complex level of care. In order for a medication error to be reported, a nurse must possess the knowledge on what is a medication error and how to report it and feel supported in the reporting process. In order to be able to recognize when an error has taken place, definitions are important, as they vary across the nation from one facility to the next. Being aware of a facility's definition is important to be able comply with safety standards as a critical care nurse.

Hospital policies offer definitions and established guidelines that are put into place to help nurses make decisions and provide an established standard of care. Knowledge of hospital policy is equally important for error recognition and to be current on nursing practices regarding medication safety and medication error reporting. Hospital policies help nurses know what is expected behavior at their facility regarding medication errors and reporting. It is also important for nurses to have knowledge and education 
regarding the event reporting system where they work. The usability of the system should be explored/reviewed periodically in order to assess whether nurses are able to access this and easily complete reports when they encounter medication errors. Whether selfreporting or not, accessible, anonymous reporting is crucial to safety culture and usability is key to making error reporting ubiquitous in the healthcare system.

Overall, the results of this survey-based project, demonstrated that there exist gaps in the knowledge base regarding hospital's adopted definition of a medication error and written policies regarding medication errors and reporting of these. Providing enhanced education aimed at closing these gaps can guide critical care nurses to confidently and consistently recognize/identify and report actual and potential medication errors.

\section{Limitations}

Several limitations of the study were identified. The main limitation of this study was that no demographic data on years of service was gathered. The literature implies that experience and knowledge prove to be safest in nursing. New nurses and those with less experience are more likely to encounter a medication error. This particular group of respondents seemed to be very knowledgeable regarding medication errors and reporting. It would have been beneficial to see the skills mix of the group.

The survey itself is self-limiting as far as its content. In the interest of time and participation, the questions were minimized. A total of ten short and succinct questions were used to explore nurses' knowledge base. It was also priority to have all questions on one, single sided paper. It is difficult to engage this particular population of nurses as they are usually a highly sought-after group for surveys and inquiries. They are also very 
busy and engaged in highly complex patient care situations throughout their shifts.

Critical care nurses' free time is scarce so making the survey as quick and simple to engage the nurses was a key point to the success of this project. This also created the potential for selection bias. Respondents were asked to self-identify as critical care nurses only in order to participate. The content created the possibility that nurses who had more knowledge or awareness of medication errors and patient safety may have been more likely to participate than those that did not. This group of respondents may not be representative of the general critical care nurse population at this facility.

The questions themselves were direct, to the point and short, but left more content/ details that could be further explored in a lengthier format. For example, why had a majority of nurses not read the hospital policy, despite knowing how to find it? What kind of education/training did the nurses received regarding medication error prevention strategies, did they find it useful, was it a one-time educational program or is this annual education given by the subject facility? Do the nurses know how to assess harm from a medication error according to hospital policy? What is it that the nurses find difficult or time consuming about reporting medication errors related to the hospital's reporting system? The percentage of nurses who believe reporting a medication error will result in disciplinary action was also not further explored. Have they ever experienced being disciplined after reporting, and how does this affect their other responses? How does disciplinary action impact their intentions to report medication errors? The nurses' perceptions of just culture at their facility was not explored in a deeper manner and couldn't have been in such a quick abbreviated survey format. 
Further testing and analysis of the survey tool used would be required to establish reliability and validity. The results of this study were exploratory findings that lay a widespread foundation for future research regarding medication errors, reporting and nurses' role in medication safety and culture within critical care settings. 


\section{Recommendations and Implications for Advanced Nursing Practice}

The results of this project demonstrated that knowledge gaps exist among critical care nurses related to definition, recognition and hospital policy procedures with regard to medication errors. Nurses indicated with their responses a lack of education and/or knowledge of the facility's adopted formal definition of a medication error as well as the policies regarding medication error reporting. They also indicated that there is still a belief among some nurses that the culture is still punitive regarding medication errors. The critical care setting is highly complex, runs at a rapid pace and patients are the most vulnerable to poor outcomes when an error is made. Providing enhanced education and providing additional institutional support may give critical care nurses the tools to apply evidenced-based interventions to their workflow that create greater awareness of medication errors and reporting practices. The ultimate goal or desired result of enhanced educational programs would be an increase in transparency regarding medication errors in critical care units, less medication errors, increased reporting of near miss events, improved patient outcomes/satisfaction, safer medication management, shorter/safer ICU stays, and increased ICU nurse confidence and satisfaction.

The Advanced Practice Registered Nurse (APRN) has a central and pivotal role in medication safety as a nurse educator and patient advocate. APRNs can affect and influence systems by adopting and supporting evidenced based practices, policies and procedures for safe medication management. They can also encourage adherence and bring awareness to established guidelines and written hospital policies regarding medication errors.

The APRN is able to collaborate and establish a team dynamic with other providers and healthcare disciplines to cultivate a culture of safety in order to increase 
and normalize reporting of medication errors and near miss events among bedside providers. Team collaboration between APRNs, critical care nurses, doctors, pharmacists and nursing leadership is essential to creating just culture that supports a non-punitive systems approach to addressing medication errors. While serving as a leader, the APRN can design and implement programs, projects and strategies for supporting and enhancing the culture of safety within their facility. As an educator, the APRN can develop and recommend educational efforts for critical care nurses to bridge knowledge gaps regarding medication errors and teach strategies to prevent medication errors.

All providers must be included in education and guidance regarding medication errors and reporting because they are at the "sharp end" of errors where they can make the most impact on prevention management. The APRN may function as an innovative leader especially in the critical care setting where patients are most vulnerable. An APRN can develop evidence-based educational programs, policies and guidelines to increase medication safety and provide guidance to increase the number and consistency of nearmiss event reporting through enhanced education of critical care nurses.

The APRN can conduct further research and scholarly writing regarding safer medication management systems, investigate barriers to medication error reporting and propose new and innovative methods for measuring patient safety and quality of care.

APRNs can also work to use their expertise to influence and educate at the community, state and national level. Medical errors are a national dilemma that plagues the medical system within which APRNs function as crucial change agents. APRNs can work to establish nonpunitive guidelines to address medical and medication errors, work with local and federal agencies or leaders to protect and advocate for the safety of all 
patients in critical care units using a systems approach based on the best evidence.

Establishing a collaborative partnership with stakeholders including patients, families and providers can help make the APRN a central role in bringing forth innovative change, establish models of care that put safety first and hold providers accountable for their errors while at the same time making transparency and learning key principles of medication safety. 


\section{References}

Agency for Healthcare Research and Quality [AHRQ]. (2008). The patient safety and quality improvement act of 2005 [Content last reviewed October 2014].

Rockville, MD. Retrieved July 6, 2019, from

https://www.ahrq.gov/policymakers/psoact.html

Agency for Healthcare Research and Quality [AHRQ]. (2013). Patient safety organizations program. [Content last reviewed March 2018]. Rockville, MD. Retrieved August 6, 2019, from https://www.ahrq.gov/cpi/about/otherwebsites/pso.ahrq.gov/index.html

Agency for Healthcare Research and Quality [AHRQ]. (2019). National score card on hospital-acquired conditions: Updated baseline rates and preliminary results 20142017. Rockville, MD. https://www.ahrq.gov/hai/pfp/2014-final.html

Ajzen, I. (1991). The theory of planned behavior. Organizational behavior and human decision processes, 50(2), 179-211.

Ajzen, I., \& Fishbein, M. (1980). Understanding attitudes and predicting social behavior. [ProQuest eBook Central version]. Englewood Cliffs, NJ: Prentice Hall. Retrieved from: https//ebookcentral.proquest.com/lib/ric-ebooks

Ajzen, I., \& Fishbein, M. (2005). The influence of attitudes on behavior. In D. Albarracin, B.T. Jonson, \& M. P. Zanna (Eds.), Handbook of attitudes and attitude change: Basic principles (pp. 173-221). Mahwah, NJ: Lawrence Erlbaum Associates.

Aspden, P., Wolcott, J. A., Bootman, J. L., \& Cronenwett, L. R. (Eds.). (2007). Preventing medication errors. Washington, DC: National Academies Press. Retrieved from https://www.nap.edu/catalog/11623/preventing-medication-errors 
Bannow, T. (2014, December 4). Error at St. Charles Bend results in patient's death. The Bulletin. Retrieved from https://www.bendbulletin.com

Barach, P., Small, S.D. (2000). Reporting and preventing medical mishaps: Lessons from non-medical near miss reporting systems. BMJ, (320), 759-763.

Barker, K. N., Flynn, E. A., Pepper, G. A., Bates, D. W., \& Mikeal, R. L. (2012). Medication errors observed in 36 healthcare facilities. Archives of Internal Medicine, 162(16), 1897-1903. http://dx.doi.org/10.1001/archinte.162.16.1897

Bates, D. W. (2000). Using information technology to reduce rates of medication errors in hospitals. $B M J, 320(7237), 788-791$. http://dx.doi.org/10.1136/bmj.320.7237.788

Bayazidi, S., Zarezadeh, Y., Zamanzadeh, V., \& Parvan, K. (2012). Medication error reporting rate and its barriers and facilitators among nurses. Journal of Caring Sciences, 1(4), 231.

Berdot, S. N., Sabatier, B., Gillaizeau, F., Caruba, T., Prognon, P., \& Durieux, P. (2012). Evaluation of drug administration errors in a teaching hospital. BMC Health Services Research, 12(60), 1-8. http://dx.doi.org/10.1186/1472-6963-12-60

Calabrese, A. D., Erstad, B. L., Brandl, K., Barletta, J. F., Kane, S. L., \& Sherman, D. S. (2001). Medication administration errors in adult patients in the ICU. Intensive Care Medicine, 27, 1592-1598. http://dx.doi.org/10.1007/s001340101065

Carayon, P., Wetterneck, T. B., Cartmill, R., Blosky, M. A., Brown, R., Kim, R., ... Walker, J. (2014, February 28). Characterizing the complexity of medication safety using a human factors approach: An observational study in two intensive 
care units. BMJ Quality \& Safety, 23(1), 56-65. http://dx.doi.org/10.1136/bmjqs2013-001828

Classen, D. C., Resar, R., Griffin, F., Federico, F., Frankel, T., Kimmel, N., ... James, B. C. (2011). Global Trigger Tool shows that adverse events in hospitals may be ten times greater than previously measured. Health Affairs, 30(4), 581-587. http://dx.doi.org/10.1377/hlthaff.2011.0190

Cohen, M. R. (Ed.). (2007). Medication Errors (2nd ed.). Washington, DC: American Pharmacist Association.

Corwin, G. S., Mills, P. D., Shanawani, H., \& Hemphill, R. R. (2017). Root case analysis of ICU adverse events in the Veterans Health Administration. Joint Commission Journal on Quality \& Patient Safety, 43(11), 580-590. http://dx.doi.org/10.1016/j.jcjq.2017.04.009

Dirik, H. F., Samur, M., Seren Intepeler, S., \& Hewison, A. (2019). Nurses' identification and reporting of medication errors. Journal of clinical nursing, 28(5-6), 931-938.

DeYoung, J.L., VanderKool, M. E., \& Barletta, J. F. (2009). Effect of bar-code assisted medication administration on medication error rates in an adult medical intensive care unit. American Journal of Health-System Pharmacy, 66(12), 1110-1115. doi: 10.2146/ajhp080355

Durham, M. L., Suhayda, R., Normand, P., \& Jankiewicz, A. (2016). Reducing medication administration errors in acute and critical care. The Journal of Nursing Administration, 46(2), 75-81. http://dx.doi.org/10.1097/NNA.0000000000000299 
Eisenhauer, L. A., Hurley, A. C., \& Dolan, N. (2007). Nurses' reported thinking during medication administration. Journal of Nursing Scholarship, 39(1), 82-87. http://dx.doi.org/10.1111/j.1547-5069.2007.00148.x

Evans, S. M., Berry, J. G., Smith, B.J., Esterman, A., Selim, P., O’Shaughnessy, J., \& DeWit, M. (2006). Attitudes and barriers to incident reporting: A collaborative hospital study. Quality \& Safety in Health Care, 15(1), 39-45. http://dx.doi.org/https://dx.doi.org/10.1136\%2Fqshc.2004.012559

Farley, D. O., Champagne, S., Jain, A. K., Battles, J. B., Munier, W. B., \& Loeb, J. M. (2008). Adverse event reporting practices by US hospitals: Results of a national survey. Quality \& Safety in Health Care, 17, 416-423. http://dx.doi.org/10.1136/qshc.2007.024638

Fishbein, M., \& Ajzen, I. (2009). Predicting and changing behavior: The reasoned action approach. [ProQuest Ebook Central]. Retrieved from https://ebookcentral.proquest.com/lib/ric-ebooks

Flynn, E. A., Barker, K. N., Pepper, G. A., Bates, D. W., \& Mikeal, R. L. (2002). Comparison of methods for detecting medication errors in 36 hospitals and skilled-nursing facilities. American Journal of Health-System Pharmacy, 59(5), 436-446. https://doi.org/10.1093/ajhp/59.5.436

Fox, B. I., Pedersen, C. A., \& Gumpper, K. F. (2015). ASHP national survey on informatics: Assessment of the adoption and use of pharmacy informatics in U.S. hospitals-2013. American Journal of Health-System Pharmacy, 72(8), 636-655. https://doi.org/10.2146/ajhp140274 
Gandhi, T. K., Seger, D. L., \& Bates, D. W. (2000). Identifying drug safety issues: From research to practice. International Journal for Quality in Health Care, 12(1), 6976. http://dx.doi.org/10.1093/intqhe/12.1.69

Giuliano, K. K. (2018). Intravenous smart pumps: usability issues, intravenous medication administration error, and patient safety. Critical Care Nursing Clinics, $30(2), 215-224$.

Grasso, B. C., Genest, R., Jordan, C. W., \& Bates, D. W. (2003). Use of chart and record reviews to detect medication errors in a state psychiatric hospital. Psychiatric Services, 54, 677-681. http://dx.doi.org/10.1176/appi.ps.54.5.677

Grissinger, M., \& Globud, N.J. (2004). How technology affects your risk of medication errors. Nursing. 34(1), 36-41

Hajibabaee, F., Joolaee, S., Peyravi, H., Alijany-Renany, H., Bahrani, N., \& Haghani, H. (2014). Medication error reporting in Tehran: a survey. Journal of nursing Management, 22(3), 304-310.

Halbesleben, J. R., Savage, G. T., Wakefield, D. S., \& Wakefield, B. J. (2010). Rework and workarounds in nurse medication administration process: Implications for work processes and patient safety. Health Care Management Review, 35(2), 124133. doi: 10.1097/HMR.0b013e3181d116c2

Hammoudi, B. M., Ismaile, S., \& Abu Yahya, O. (2018). Factors associated with medication administration errors and why nurses fail to report them. Scandinavian Journal of Caring Sciences, 32(3), 1038-1046.

http://dx.doi.org/10.1111/scs.12546 
Hartnell, N., MacKinnon, N., Sketris, I., \& Fleming, M. (2012). Identifying, understanding and overcoming barriers to medication error reporting in hospitals: a focus group study. BMJ Quality \& Safety, 21(5), 361-368. Doi: 10.1136/bmjqs2011-000299

Hazell, L., \& Shakir, S. A. (2006). Under-reporting of adverse drug reactions. Drug Safety, 29(5), 385-396. http://dx.doi.org/10.2165/00002018-200629050-00003

Helmons, P. J., Wargel, L. N., \& Daniels, C. E. (2009). Effect of bar-code-assisted medication administration on medication administration errors and accuracy in multiple patient care areas. American Journal of Health-System Pharmacy, 66(13), 1202-1210. http://dx.doi.org/10.2146/ajhp080357

Herout, P. M., \& Erstad, B. L. (2004). Medication errors involving continuously infused medications in a surgical intensive care unit. Critical Care Medicine, 32(2), 428432. http://dx.doi.org/10.1097/01.CCM.0000108876.12846.B7

Hicks, R. W., \& Becker, S. C. (2006). An overview of intravenous-related medication administration errors as reported to MEDMARX, a national medication errorreporting program. Journal of Infusion Nursing, 29(1), 20-27.

Hicks, R. W., Cousins, D. D., \& Williams, R. L. (2004). Selected medication-error data from USP's MEDMARX program for 2002. American Journal of Health-System Pharmacy, 61(10), 993-1000. https://doi.org/10.1093/ajhp/61.10.993

Hughes, R. G., \& Blegen, M. A. (2008). Medication Administration Safety. In Patient safety and quality: An evidence-based handbook for nurses (Ch. 37). [NCBI Bookshelf]. Retrieved from https://www.ncbi.nlm.nih.gov/books 
Husch, M., Sullivan, C., Rooney, D., Barnard, C., Fotis, M., Clarke, J., \& Noskin, G. (2005). Insights from the sharp end of intravenous medication errors: implications for infusion pump technology. BMJ Quality \& Safety, 14(2), 80-86. http://dx.doi.org/10.1136/qshc.2004.011957

Institute for Safe Medication Practices. (2017). 2018-2019 Targeted medication safety best practices for hospitals. Retrieved from https://www.ismp.org/guidelines/bestpractices-hospitals

Institute for Safe Medication Practices. (2019). About us. Retrieved from https://www.ismp.org/about

Institute of Medicine. (2001). Using information technology. In, Crossing the quality chasm: A new health system for the 21st century (pp. 164-180). Washington, DC: National Academies Press. Retrieved from http://dx.doi.org/10.17226/10027

Institute of Medicine. (2004). Patient safety: Achieving a new standard for care. Washington, DC: National Academies Press. https://doi.org/10.17226/10863

Joolace, S., Hajibabaee, F., Peyrovi, H., Haghani, H., \& Bahrani, N. (2011). The relationship between incidence and report of medication errors and working conditions. International Nursing Review, 58(1), 37-44. http://dx.doi.org/10.1.1.461.9597\&rep=rep1\&type=pdf

Kane-Gill, S. L., Kirisci, L., Verrico, M. M., \& Rothschild, J. M. (2012). Analysis of risk factors for adverse drug events in critically ill patients. Critical Care Medicine, 40(3), 823-828. http://dx.doi.org/10.1097.CCM.0b0013e318236f473

Keane, K. (2014). Reducing medication errors by educating nurses on bar code technology. MedSurg Nursing, 23(5), S1-S1 
Keohane, C. A., Bane, A. D., Featherstone, E., Hayes, J., Woolf, S., Hurley, A., ... Poon, E. G. (2008). Quantifying Nursing Workflow in Medication Administration. The Journal of Nursing Administration, 38(1), 19-26. http://dx.doi.org/10.1097/01.NNA.0000295628.87968.bc

Keers, R. N., Williams, S. D., Cooke, J., \& Ashcroft, D. M. (2013). Causes of medication administration errors in hospital: A systematic review of quantitative and qualitative evidence. Drug Safety, 35(11), 1045-1067. http://dx.doi.org/10.1007/s40264-013-0090-2

Keers, R. N., Williams, S. D., Cooke, J., \& Ashcroft, D. M. (2015). Understanding the causes of intravenous medication administration errors in hospitals: A qualitative critical incident study. BMJ Open, 5(e005948), 1-9. http://dx.doi.org/10.1036/bmjopen-2014-005948

Kelman, B. (2019). Vanderbilt ex-nurse indicted on reckless homicide after deadly medication swap. The Tennessean. Retrieved from https://www.tennessean.com

Kiekkas, P., Karga, M., Lemonidou, C., Aretha, D., \& Karanikolas, M. (2011). Medication errors in critically ill adults: a review of direct observation evidence. American Journal of Critical Care, 20(1), 36-44.

Kohn, L. T., Corrigan, J. M., \& Donaldson, M. S. (Eds.). (1999). To err is human: Building a safer health system. Washington, DC: National Academies Press. Retrieved from https://www.nap.edu/search/?term=to+err+is+human

Kopp, B. J., Erstad, B. L., Allen, M. E., Theodorou, A. A., \& Priestly, G. (2006). Medication errors and adverse drug events in an intensive care unit: Direct 
observation approach for detection. Critical Care Medicine, 34(2), 415-425. http://dx.doi.org/10.1097/01.CCM.0000198106.54306.D7

Koppel, R., Wetterneck, T., Telles, J. L., \& Karsh, B. T. (2008). Workarounds to barcode medication administration systems: their occurrences, causes, and threats to patient safety. Journal of the American Medical Informatics Association, 15(4), 408-423. doi: 10.1197/jamia.M2616.

Kuhns, M., \& McEwen, M. (2011). Theories from the behavioral sciences. In M. McEwen \& E. M. Wills, Theoretical basis for nursing (3rd ed. (pp. 290-295). Philadelphia, PA: Lippincott Williams \& Wilkins.

Landrigan, C. P., Parry, G. J., Bones, C. B., Hackbarth, A. D., Goldmann, D. A., \& Sharek, P. J. (2010). Temporal trends in rates of patient harm resulting from medical care. The New England Journal of Medicine, 363(22), 21242134.

Latif, A., Rawat, N., Pustavoitau, A., Pronovost, P. J., \& Pham, J. C. (2013). National study on the distribution, causes, and consequences of voluntarily reported medication errors between the ICU and non-ICU settings. Critical Care Medicine, 41(2), 389-398. http://dx.doi.org/10.1097/CCM.0b013e318274156a

Leape, L. (1994). Error in medicine. JAMA, 272(23), 1851-1857. http://dx.doi.org/10.1001/jama.1994.03520230061039

Levinson, D. R. (2008). Adverse events in hospitals: Case study of incidence among Medicare beneficiaries in two selected counties (OEI-06-08-00220). Retrieved from U.S. Department of Health and Human Services, Office of Inspector General website: https://oig.hhs.gov/reports-and-publications/oei/h.asp 
Levinson, D. R. (2010). Adverse events in hospitals: National incidence among Medicare beneficiaries (OEI-06-09-00090). Retrieved from U.S. Department of Health and Human Services, Office of Inspector General website: https://oig.hhs.gov/oei/reports/oei-06-09-00090.pdf

Lisby, M., Nielsen, L. P., \& Mainz, J. (2005). Errors in the medication process: Frequency, type and potential clinical consequences. International Journal for Quality in Health Care, 17(1), 15-22. http://dx.doi.org/10.1093/intqhc/mzi015

MacFie, C. C., Baudouin, S. V., \& Messer, P. B. (2016). An integrative review of drug errors in critical care. Journal of the Intensive Care Society, 17(1), 63-72. http://dx.doi.org/10.1177/1751143715605119

Makary, M. A., \& Daniel, M. (2016). Medical error - the third leading cause of death in the US. $B M J, 353$. http://dx.doi.org/10.1136/bmj.i2139

Mayo, A. M., \& Duncan, D. (2004). Nurse perceptions of medication errors: What we need to know for patient safety. Journal of Nursing Care Quality, 19(3), 209-217. Retrieved from http://web.b.ebscohost.com.ric.idm.oclc.org

Milch, C. E., Salem, D. N., Pauker, S. G., Lundquist, T. G., Kumar, S., \& Chen, J. (2005). Voluntary electronic reporting of medical errors and adverse events: An analysis of 92,547 reports from 26 acute care hospitals. Journal of General Internal Medicine, 21, 165-170. http://dx.doi.org/10.1111/j.15251497.2006.00322.x

Montano, D. E., \& Kasprzyk, D. (2002). The theory of reason action and the theory of planned behavior. In K. Glanz, F. M. Lewis, \& B. K. Rimer, Health behavior and 
health education: Theory, research and practice (3rd ed., pp. 67-96). San Francisco, CA: John Wiley \& Sons.

Moyen, C. E., \& Stelfox, H. T. (2008). Clinical review: Medication errors in critical care. Critical Care, 12(2), 1-7.

Murff, H. J., Patel, V. L., Hripcsak, G., \& Bates, D. W. (2003). Detecting adverse events for patient safety research: A review of current methodologies. Journal of Biomedical Informatics, 36, 131-143. http://dx.doi.org/10.1016/j.jbi.2003.08.003

National Coordinating Council for Medication Error Reporting and Prevention. (2015). Two decades of coordinating medication safety efforts. Retrieved from https://www.nccmerp.org/sites/default/files/20_year_report.pdf

National Quality Forum. (2011). Serious reportable events in healthcare - 2011 update: A consensus report. Retrieved from http://www.qualityforum.org/topics/sres/serious_reportable_events.aspx

Nebeker, J.R., Hoffman, J.M., Weir, C.R., Bennett, C.L. \& Hurdle, J.F. (2005). High rates of adverse events in a highly computerized hospital. Archives of Internal Medicine, 165, 1111-1116. http://dx.doi.org10.1001/archinte.165.10.1111

Nuckols, T. K., Bell, D. S., Liu, H., Paddock, S. M., \& Hilborne, L. H. (2007). Rates and types of events reported to established incident reporting systems in two US hospitals. Quality \& Safety in Health Care, 16, 164-168. http://dx.doi.org/10.1136/qshc.2006.019901

O'Byrne, N., Kozub, E. I., \& Fields, W. (2016). Reducing continuous intravenous medication errors in an intensive care unit. Journal of nursing care quality, 3l(1), $13-16$. 
Ohashi, K., Dalleur, O., Dykes, P. C., \& Bates, D. W. (2014). Benefits and risks of using smart pumps to reduce medication error rates: A systematic review. Drug Safety, 37, 1011-1020. http://dx.doi.org/10.1007/s40264-014-0232-1

Pederson, C. A., \& Gumpper, K. F. (2008). ASHP national survey on informatics: Assessment of the adoption and use of pharmacy informatics in U.S. hospitals2007. American Journal of Health-System Pharmacy, 65(23), 2244-2264. http://dx.doi.org/https://doi.org/10.2146/ajhp080488

Phillips, M. A. (2002). Voluntary reporting of medication errors. American Journal of Health-System Pharmacy, 59, 2326-2328. http://dx.doi.org/https://doi.org/10.1093/ajhp/59.23.2326

Pinkney, S., Trbovich, P., Fan, M., Rothwell, S., Cafazzo, J. A., \& Easty, A. (2010). Do Smart pumps actually reduce errors? Biomedical Instrumentation \& Technology, 44, 64-69. http://dx.doi.org/10.2345/0899-8205-44.s1.64

Poon, E. G., Keohane, C. A., Yoon, C. S., Ditmore, M., Bane, A., Levtzion-Korach, O., ... Gandhi, T. K. (2010). Effect of barcode technology on the safety of medication administration. New England Journal of Medicine, 362, 1698-1707. http://dx.doi.org/10.1056/NEJMsa0907115

Quantros. (2009). MEDMARX adverse event reporting. Retrieved from https://www.medmarx.com

Reason, J. (1990). Human Error. New York, NY: Cambridge University Press.

Reason, J. (1995). Understanding adverse events: Human factors. BMJ Quality \& Safety 4(2), 80-89. Retrieved from: 
https://www.ncbi.nlm.nih.gov/pmc/articles/PMC1055294/pdf/qualhc000160008.pdf

Reason J. (2000). Human error: Models and management. BMJ (Clinical research ed.), 320(7237), 768-770. doi:10.1136/bmj.320.7237.768

Reason, J. (2001). A systems approach to organizational error. Ergonomics, 38(8), $1708-1721$.

Rose, B.J. (2016). Introduction to FDA's MedWatch adverse event reporting program. [Webinar] Retrieved from https://www.fda.gov/media/95928/download Rothschild, J. M., Keoane, C. A., Cook, E. F., Orav, E. J., Burdick, E., Thompson, S., ... Bates, D. W. (2005a). A controlled trial of smart infusion pumps to improve medication safety in critically ill patients. Critical Care Medicine, 33(3), 533-540. http://dx.doi.org/10.1097/01.CCM.0000155912.73313.CD

Rothschild, J. M., Landrigan, C. P., Cronin, J. W., Kaushal, R., Lockley, S. W., Burdick, E., ... Bates, D. W. (2005b). The critical care safety study: The incidence and nature of adverse events and serious medical errors in intensive care. Critical Care Medicine, 33, 1694-1700. http://dx.doi.org/10.1097/01.CCM.0000171609.91035.BD

Russo, M., Buonocore, F., \& Ferrara, M. (2015). Motivational mechanisms influencing error reporting among nurses. Journal of Managerial Psychology, 30(2), 118-132. Doi: 10.1108/JMP-02-2013-0060

Rutledge, D. N., Retrosi, T., \& Ostrowski, G. (2018). Barriers to medication error reporting among hospital nurses. Journal of Clinical Nursing, 27, 1941-1949. http://dx.doi.org/10.1111.jocn.14335 
Sanghera, I. S., Franklin, B. D., \& Dhillon, S. (2007). The attitudes and beliefs of healthcare professionals on the causes and reporting of medication errors in a UK intensive care unit. Anesthesia, 62(1), 53-61. http://dx.doi.org/https://doi.org/10.1111/j.1365-2044.2006.04858.x

Soydemir, D., Seren Intepeler, S., \& Mert, H. (2017). Barriers to medical error reporting for physicians and nurses. Western Journal of Nursing Research, 39(10), 13481363.

Stergiopoulos, S., Brown, C. A., Felix, T., Grampp, G., \& Getz, K. A. (2016). A survey of adverse event reporting practices among US healthcare professionals. Drug Safety, 39(11), 1117-1127. https://doi.org/10.1007/s40264-016-0455-4

Sujan, M. (2015). An organization without a memory: a qualitative study of hospital staff perceptions on reporting and organizational learning for patient safety. Reliability Engineering \& System Safety, 144, 45-52. http://dx.doi.org/10.1016/j.ress.2015.07.011

Taxis, K., \& Barber, N. (2003). Ethnographic study of incidence and severity of intravenous drug errors. British Medical Journal, 326, 684. http://dx.doi.org/https://dx.doi.org/10.1136\%2Fbmj.326.7391.684

The Joint Commission. (2018). Developing a reporting culture: Learning from close calls and hazardous conditions [Sentinel event alert]. Retrieved from https://www.jointcommission.org/assets/1/18/SEA_60_Reporting_culture_FINAL .pdf 
The Joint Commission. (2019). Sentinel events. Comprehensive Accreditation Manual for Hospitals. [E-dition July 1, 2019 Release.] Retrieved July 6, 2019 from https://www.jointcommission.org/sentinel_event_policy_and_procedures/

Truitt, E., Thompson, R., Blazey-Martin, D., NiSai, D., \& Salem, D. (2018). Effect of the implementation of barcode technology and an electronic medication administration record on adverse drug events. Hospital Pharmacy, 51, 474-483. http://dx.doi.org/https://dx.doi.org/10.1310\%2Fhpj5106-474

U.S. Food \& Drug Administration. (2018). Reporting Serious Problems to FDA. MedWatch: The FDA Safety Information and Adverse Reporting Program website. Retrieved from: https://www.fda.gov/safety/medwatch-fda-safetyinformation-and-adverse-event-reporting-program on June 30, 2019.

Uribe, C. L., Schweikhart, S. B., Pathak, D. S., \& Marsh, G. B. (2002). Perceived barriers to medical-error reporting: An exploratory investigation. Journal of Healthcare Management, 47(4), 263-280. Retrieved from http://web.a.ebscohost.com.ric.idm.oclc.org/ehost

Valentin, A., Capuzzo, M., Guidet, B., Moreno, R., Metnitz, B., Bauer, P., \& Metnitz, P. (2009, March 13). Errors in administration of parenteral drugs in intensive care units: Multinational prospective study. $B M J, 338$. http://dx.doi.org/https://doi.org/10.1136/bmj.b814

Wakefield, B. J., Blegen, M. A., Uden-Holman, T., Vaughn, T., Chrischilles, E., \& Wakefield, D. S. (2001). Organizational culture, continuous quality improvement, and medication administration error reporting. American Journal of Medical 
Quality, 16(4), 128-134. Retrieved from

http://web.b.ebscohost.com.ric.idm.oclc.org

Wakefield, B. J., Uden-Holman, T., \& Wakefield, D. S. (2005). Development and validation of the administration error reporting survey. Advances in Patient Safety, 4, 475-489. Retrieved from https://apps.dtic.mil/dtic/tr/fulltext/u2/a434734.pdf

Wakefield, B., Wakefield, D. S., \& Uden-Holden, T. (2000). Improving medication administration error reporting systems. Ambulatory Outreach, 16-20.

Wakefield, D. S., Wakefield, B., Uden-Holman, T., \& Blegen, M. A. (1996). Perceived barriers in reporting medication administration errors. Best Practices and Benchmarking in Healthcare, 1(4), 191-197.

Wakefield, D., Wakefield, B., Uden-Holman, T., Borders, T., Blegen, M., \& Vaughn, T. (1999). Understanding and comparing differences in reported medication administration error rates. American Journal of Medical Quality, 14(2), 73-80.

Wakefield, D., Wakefield, B., Uden-Holman, T., Borders, T., Blegen, M., \& Vaughn, T. (1999). Understanding why medication administration errors may not be reported. American Journal of Medical Quality, 14(2), 81-88.

Westbrook, J. L., Li, L., Hooper, T. D., Raban, M. Z., Middleton, S., \& Lehnbom, E. C. (2017). Effectiveness of a 'do not interrupt' bundled intervention to reduce interruptions during medication administration: A cluster randomized controlled feasibility study. BMJ Quality \& Safety, 26, 734-742.

http://dx.doi.org/10.1136/bmjqs-2016-006123 
Westbrook, J. I., Li, L., Lehnbom, E. C., Baysari, M. T., Braithwaite, J., Burke, R., ... \& Day, R. O. (2015). What are incident reports telling us? A comparative study at two Australian hospitals of medication errors identified at audit, detected by staff and reported to an incident system. International Journal for Quality in Health Care, 27(1), 1-9. Doi: 10.1093/intqhc/mzu098

Westbrook, J. L., Rob, M. I., Woods, A., \& Parry, D. (2011). Errors in the administration of intravenous medications in hospital and the role of correct procedures and nurse experience. BMJ Quality \& Safety, 2, 1027-1034. http://dx.doi.org/10.1136/bmjqs-2011-000089

Wilmer, A., Louie, K., Dodek, P., Wong, H., \& Ayas, N. (2010, July 29). Incidence of medication errors and adverse drug events in the ICU: A systematic review. Quality \& Safety in Health Care, 19(5), 1-9. http://dx.doi.org/10.1136/qshc.2008.030783

Wirtz, V., Barber, N., \& Taxis, K. (2003). An observational study of intravenous medication errors in the United Kingdom and in Germany. Pharmacy World and Science, 25(3), 104-111. https://doi.org/10.1023/A:1024009000113

Winterstein, A. G., Johns, T. E., Rosenberg, E. I., Hatton, R. C., Gonzalez-Rothi, R., \& Kanjanarat, P. (2004). Nature and causes of clinically significant medication errors in a tertiary care hospital. American Journal of Health-System Pharmacy, 61, 1908-1916. https://doi.org/10.1093/ajhp/61.18.1908 


\section{Appendix A}

\section{Reporting Medication Administration Error Survey}

1. Are you aware of the hospital's definition of a medication error?
a. Yes
b. No

2. Do you know how to find the hospital's policy on medication errors?
a. Yes
b. No

If yes, have you read it?
a. Yes
b. No

3. A near-miss event and a medication error are the same.
a. True
b. False

4. If there is no harm to the patient, an incident involving a medication does not need to be reported.
a. True
b. False

5. Have you received education/training regarding medication error prevention strategies?
a. Yes
b. No

6. Do you know how to access the hospital's event reporting system (Safety Net)?
a. Yes
b. No

7. Reporting a medication error is fast and easy.
a. True
b. False

8. Reporting a medication error will result in disciplinary action.
a. True
b. False

9. All adverse drug events should be reported via the hospital's reporting system.
a. Ture
b. False 
10. An order for a continuous infusion is entered in a patient's chart. The medication is obtained from Omnicell, scanned and is infusing at the ordered dose in $\mathrm{mcg} / \mathrm{kg} / \mathrm{min}$. An hour later, during bedside handoff, it is noticed that the medication infusion rate in $\mathrm{mL} /$ hour on the pump and the order do not match. The weight entered into the infusion pump does not match the weight documented in the electronic chart. Is this a reportable event?
a. Yes
b. No 
Appendix B

Approval letter from CNO

Rhode Island Hospital

Lifosyom. Delizering health with care.

593 Eddy Street

Prowidence, R1 02903

Tet $401444-4000$

October 2, 2019

Rhode Island Hospital Institutional Review Board

Research Administrator

Coro West 1.300

Dear Madam:

I am writing this letter in support of Registered Nurse Karina Santos's graduate program project entitled "Exploring Nurses' Knowledge of Medication Error Reporting." This project may help us improve nurses reporting of medication errors and so support a culture of safety.

Yours truly,

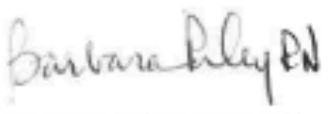

Barbara Riley, DNP, RN

Sr. Vice-President \& Chief Nursing Officer

Rhode Island Hospital \& Hasbro Children's Hospital 


\section{Appendix C}

\section{Information related to quality improvement project on nurses' knowledge of the medication error reporting process}

As part of a graduate quality improvement project, Karina Santos, Master of Nursing Science student at Rhode Island College, will be distributing a survey to explore nursing staff's knowledge about reporting of medication errors within the critical care units .Purpose of the survey; Describe survey here- short, 10 questions, multiple choice and true or false, no identifying information The survey will be available in January 2020 and located in the break room. If you are interested in participating in this project, please review the informational letter attached to the survey. If you then decide to participate, complete the survey and place it in the sealed box provided. Responses are anonymous and your decision to participate or not, is your choice. 


\section{Appendix D}

\section{Presentation to Clinical Leadership at Roundtable Meetings}

My name is Karina Santos, and I am a Master of Nursing Science student at Rhode Island College. I will be distributing a survey to explore nursing staff's knowledge about reporting of medication errors within our critical care division. The paper survey will be available in January 2020 and located in the break rooms along with an informational letter attached to the survey. If staff RNs decide to participate, they may complete the survey and place it in the sealed box provided. Responses are anonymous and it is the RN's decision to participate or not. 
Appendix E

Recruitment Flyer for Unit Bulletin Boards

\section{ARE YOU A CRITICAL CARE RN? ARE YOU CONCERNED ABOUT MEDICATION ERRORS?}
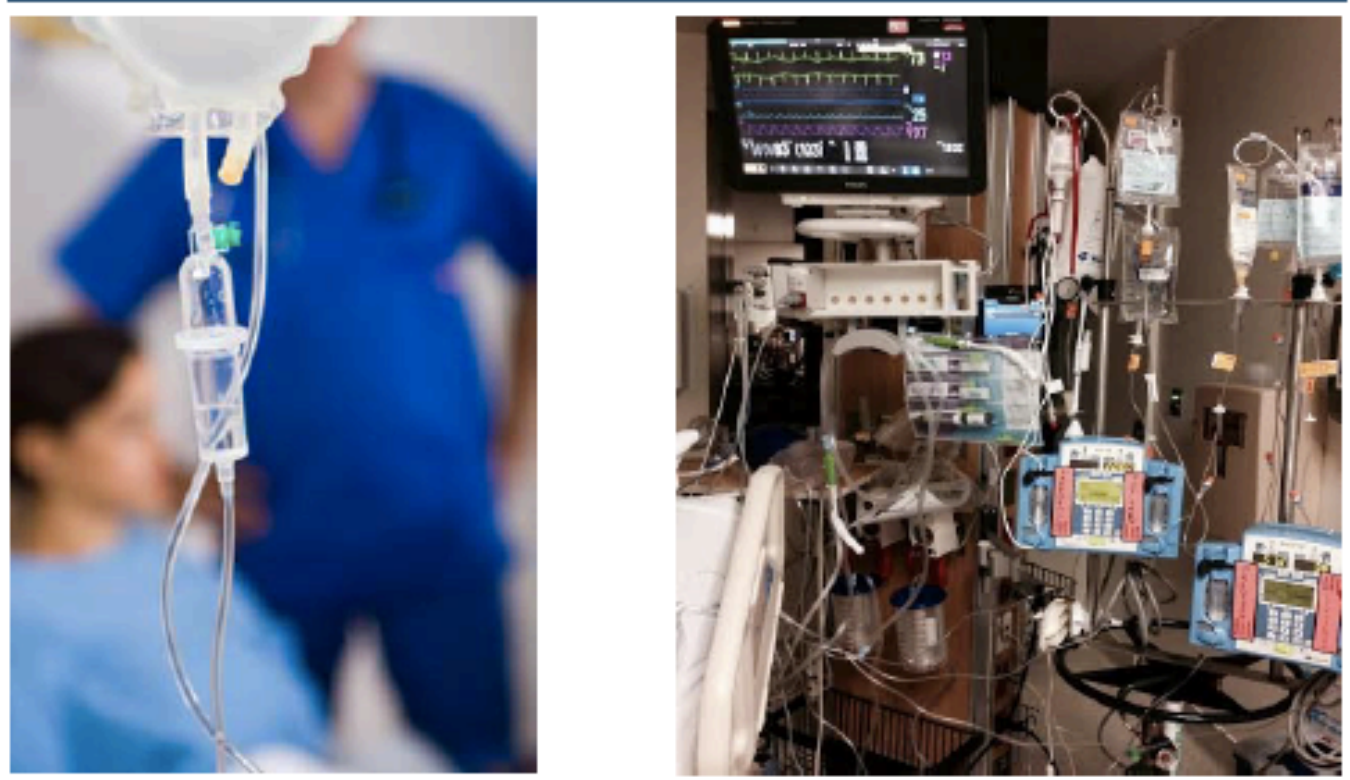

\section{Consider participating in a survey project}

The purpose of this project is to explore the knowledge base of critical care nurses regarding the reporting of medication errors. The survey is short should only take 5 minutes or less. Your participation is completely optional and anonymous

Karina Santos:|401-484-0162: Ksantos2@lifespan.org 


\section{Appendix F \\ Informational Letter}

To whom it may concern:

We would like you to consider participating in a quality improvement project titled: Exploring nurses' knowledge of medication error reporting in the critical care setting: A quality improvement project. The Principal Investigator for this project will be Joan Walsh. The purpose of this project is to explore the knowledge base of critical care nures regarding the reporting of medication errors. Participation is voluntary and if you agree to participate, it will involve the completion of an anonymous survey. The goal of this project is to identify knowledge gaps regarding medication errors and the reporting process to help guide future educational materials.

The survey will consist of 10 questions aimed at identifying how much is known about identifying and reporting medication errors and should not cause any level of discomfort. Though there are no direct benefits to participating in this project, you may increase your knowledge regarding medication errors and reporting of these. Participation in the survey is voluntary, anonymous and you may withdraw at any time. Completed surveys may be submitted in the sealed box located in your breakroom. They will be collected by the student investigator, Karina Santos and stored in a secure location. I hope you will consider participating in this quality improvement project.

If you have any questions about your rights as a research subject please feel free to call our Research Protections Office Director, Janice Muratori, at 401-444-6897. You 
may also contact Joan Walsh, Jwalsh1@lifespan.org, 401-444-6287 or Karina Santos, ksantos_9566@email.ric.edu, 401-484-0162 with any questions regarding this project.

Thank you for your participation,

Joan Walsh, DNP, APRN-CNS

Karina Santos, BSN, RN 